\title{
Active metamaterial nearly perfect light absorbers: a review [Invited]
}

\author{
Hodjat Hajian, ${ }^{1, *}$ (i) Amir Ghobadi, ${ }^{1,2}$ (i) Bayram Butun, ${ }^{1}$ (i) and Ekmel Ozbay ${ }^{1,2,3,4,5}$ \\ ${ }^{1}$ NANOTAM-Nanotechnology Research Center, Bilkent University, 06800 Ankara, Turkey \\ ${ }^{2}$ Department of Electrical and Electronics Engineering, Bilkent University, 06800 Ankara, Turkey \\ ${ }^{3}$ UNAM-Institute of Materials Science and Nanotechnology, Bilkent University, 06800 Ankara, Turkey \\ ${ }^{4}$ Department of Physics, Bilkent University, 06800 Ankara, Turkey \\ ${ }^{5}$ e-mail: ozbay@bilkent.edu.tr \\ *Corresponding author: hodjat.hajian@bilkent.edu.tr
}

Received 27 February 2019; revised 21 April 2019; accepted 21 April 2019; posted 22 April 2019 (Doc. ID 361230); published 9 July 2019

\begin{abstract}
Achieving nearly perfect light absorption from the microwave to optical region utilizing metamaterials has begun to play a significant role in photonics and optoelectronics due to their vital applications in thermal emitters, thermal photovoltaics, photovoltaics, sensing, filtering, and photodetection. However, employing passive components in designing perfect absorbers based on metamaterials and photonic crystals imposes some limits on their spectral operation. In order to overcome those limits, extensive research has been conducted on utilizing different materials and mechanisms to obtain active metamaterial light absorbers. In this review paper, we investigate the recent progress in tunable and reconfigurable metamaterial light absorbers through reviewing different active materials and mechanisms, and we provide a perspective for their future development and applications. @ 2019 Optical Society of America
\end{abstract}

https://doi.org/10.1364/JOSAB.36.00F131

\section{INTRODUCTION}

Metamaterials [1,2], the so-called artificially engineered structures, demonstrate interesting electromagnetic properties that are not found in natural materials including negative refractive index [3], electromagnetic wave cloaking, [4], inverse Doppler effect [5], lasing [6], asymmetric light transmission [7], and artificial magnetism [8]. Light absorption is another eye-catching characteristic of these artificial structures [9], and the metamaterials with nearly perfect light absorption features are referred as metamaterial nearly perfect light absorbers (MPAs). In order to realize nearly perfect absorption, reflectance is suppressed by matching the effective impedance of the metamaterial to that of the incident medium [10]. Simultaneously, transmittance may be eliminated by introducing another metallic plate acting as a mirror [11] or by using a similar mechanism in the multilayer systems. Based on these approaches, MPAs can be categorized into two types: narrowband MPAs [12-31] and broadband MPAs [32-47]. For the structures shown in panels (a) [26] and (b) [27] of Fig. 1, the narrowband nearly perfect absorptions can be obtained by planar metal-insulator-metal (MIM) metamaterials for which the top layer is either patterned [panel (a)] or unpatterned [panel (b)]. For the former structure, the support of localized and propagating surface plasmons polaritons (SPPs) is responsible for the narrowband response while, for the latter, cavity modes take the lead. As shown in Fig. 1(c) [28], MIMI systems for which the middle metallic layer is unpatterned can also provide us with a narrowband response due to the support of cavity modes and further satisfaction of the impedance-matching condition due to the presence of the top anti-reflecting dielectric layer. The support of surface lattice resonances-which are essentially intricate combinations of localized/delocalized surface plasmon resonances on diffractive orders in periodic arrays-is the physical mechanism behind the narrowband response obtained by the metamaterial shown in Fig. 1(d) [29]. For the metal-based one-dimensional photonic crystal (1D PC) depicted in Fig. 1(e), the support of Tamm plasmon polaritons is the reason for the narrowband absorption response [30]. In addition, the physical mechanism behind the narrowband absorption response of the unpatterned complementary metaloxide-semiconductor (CMOS)-compatible semiconductor-based design that is shown in Fig. 1(f) relates to the support of the cavity-like modes inside the top lossy layer [31]. On the other hand, in the patterned MIM metamaterials, by either having patches with different geometrical shapes and thereby overlapping various resonant responses of the structure [Fig. 1(g)] [35,42] or using lossy metals like Ti for the top patterned layer [Fig. 1(h)] [43], it is possible to achieve broadband nearly perfect absorption. As schematically shown in Fig. 1(i) [44], nearly perfect broadband absorption can also be obtained by using a pyramid shape arrangement of hyperbolic metamaterials/materials on a reflecting layer that leads to providing gradual satisfaction of the impedance matching condition for an incident light. Achieving broadband absorption is further possible by designing a tandem structure composed of three absorptive materials, while the overall structure 
features a graded refractive index profile to obtain a wideband antireflection property as schematically shown in Fig. 1(j) [45]. In this design, the optical resonances are strongly overlapped, leading to a broadband response. Due to the cancellation of the reflected wave using the arrangement of ultra-thin lossy metallic films in multilayer metal-dielectric metamaterials as shown schematically in Fig. 1(k) [46], it is also possible to achieve wideband nearly perfect absorption. Employing the dewetting process and the formation of nanoholes in the top metallic layer in an MIMI multilayer metamaterial, as shown in Fig. 1(k), is also a unique approach to realize lithography-free wideband MPAs [47].

After the first realization of MPAs [9], a tremendous amount of research interest has been attracted to the field of metamaterials to realize MPAs operating from the microwave to the optical spectral range [12-47]. MPAs have found numerous applications depending on their narrow- or broadband responses. The narrowband metal perfect absorbers are of particular interest in sensing [48], imaging [49], thermal emitting [50], and color filter applications [51,52]. On the other hand, their broadband counterparts have potential applications in thermal photovoltaics [53], radiative cooling [54], hot electron-based photodetectors [55], photochemistry [56], and efficient solar vapor/steam generation [57]. However, having a passive response may impose some limits on the operational bandwidth and thus the functionality of the metamaterial light absorbers. Therefore, extensive research has been performed on utilizing different materials and mechanisms to design metamaterials with active functionalities, i.e., the emergence of the field of active photonics and nanophotonics [58-61].

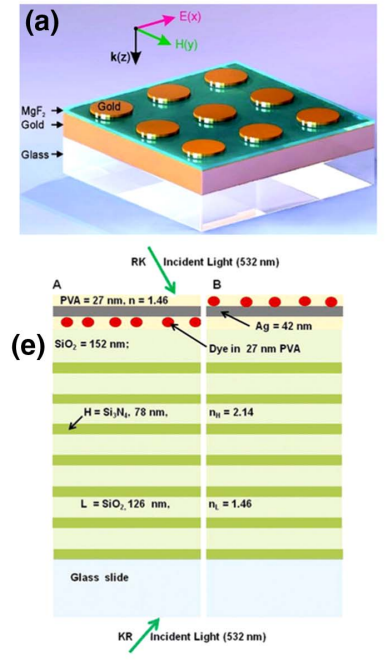

(i)

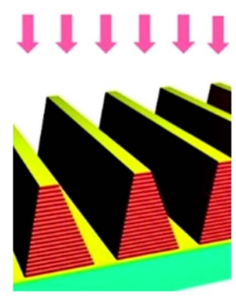

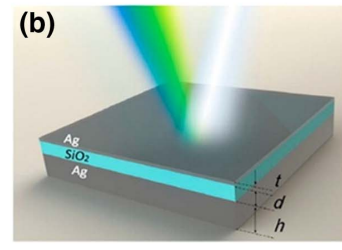

(f)

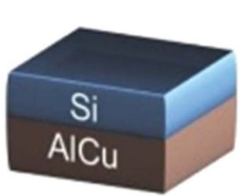

(j)

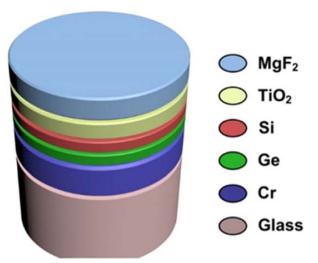

(c)

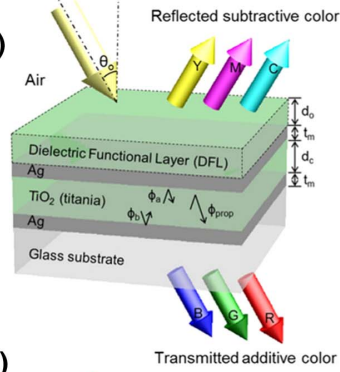

(g)

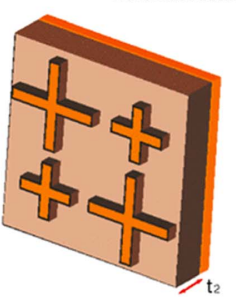

(k)

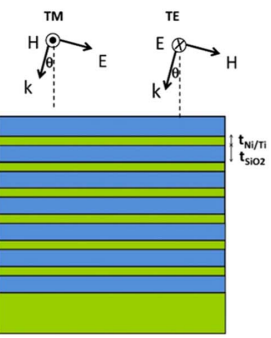

(d)

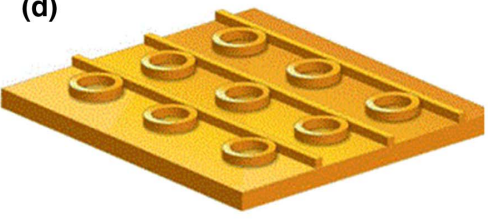

(h)

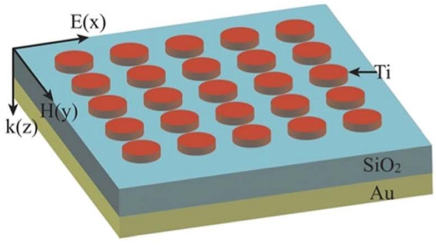

(I)

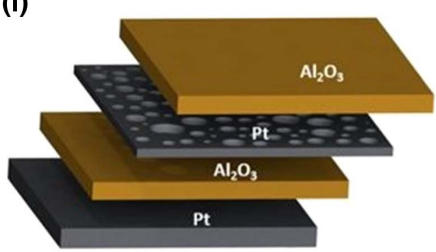

Fig. 1. Schematics of different structures that can be realized to achieve narrowband and broadband MPAs. Panels (a) [26] and (b) [27] are two MIM narrowband MPAs for which the top metallic layer is either patterned or unpatterned, respectively. Panels (c) [28] and (d) [29], respectively, illustrate unpatterned MIMI and nanoring/nanowire composite metal-based narrowband MPAs. The schematics of a one-dimensional metal-based MPA [30] and Si layer directly deposited on a reflector [31] are provided in panels (e) and (f), respectively, as other examples of unpatterned narrowband MPAs. Schematics of the broadband metamaterial perfect absorbers are shown in panels (g) to (l). Patterned MIM metamaterials using different geometrical patterning and loss materials as the top layer are provided in panels (g) [35,42] and (h) [43]. Panels (i) [44] and (j) [45] show ultrabroadband MPAs composed of sawtooth multilayer metal-dielectric and unpatterned multilayer arrangements, respectively. A multilayer metal-dielectric metamaterial perfect absorber [46] and a lithography-free MIMI MPA obtained by the dewetting process [47] are shown in panels $(\mathrm{k})$ and $(\mathrm{l})$, respectively. Notice that the mentioned MPAs operate within the visible and near-infrared ranges $(\lambda<5 \mu \mathrm{m})$; however, by appropriate designs and taking suitable lossy materials (including both plasmonic and phononic materials), it is possible to extend the range of operation to the MIR and FIR regions. (a) Reprinted with permission from Ref. [26]. Copyright 2010 American Chemical Society. (b) Reprinted with permission from Ref. [27]. Copyright 2015 American Chemical Society. (c) Reprinted from Ref. [28]. Copyright 2016 Springer Nature. (d) Reprinted with permission from Ref. [29]. Copyright 2014 American Chemical Society. (e) Reprinted with permission from Ref. [30]. Copyright 2014 American Chemical Society. (f) Reprinted with permission from Ref. [31]. Copyright 2018 John Wiley and Sons. (g) Reprinted with permission from Ref. [42]. Copyright 2011 American Physical Society. (h) Reprinted from Ref. [43]. Copyright 2016 Springer Nature. (i) Reprinted with permission from Ref. [44]. Copyright 2012 American Chemical Society. (j) Reprinted with permission from Ref. [45]. Copyright 2016 American Chemical Society. (k) Reprinted with permission from Ref. [46]. Copyright 2016 Optical Society of America. (l) Reprinted from Ref. [47]. Copyright 2017 Springer Nature. 
Active, tunable, or reconfigurable materials have been found as important building blocks in designing metamaterials with modifiable optical responses. Tunable waveguides [62], modulators [63], frequency selective surfaces [64], and metalenses [65] can be mentioned as some examples of those metamaterials. There are several mechanisms and approaches that may be taken to achieve active optical responses. Employing active materials with electrically [66-68], thermally [69,70], optically [71,72], and magnetically $[73,74]$ tunable features as the building block components of the metamaterials can be one approach. Taking advantage of stretchable materials [75-77] and micro- or nanoelectromechanical systems (M-NEMS) [78-83] is another way of achieving active metamaterials. Recently, the mentioned mechanisms have been deeply examined by Jiang et al. [84] in a review paper on the investigation of active plasmonics. In a different point of view, Nemati et al. [85] conducted an in-depth examination on tunable and reconfigurable metasurfaces and metadevices according to the different types of active materials being used in those structures. There are several different categories of active materials that are available in nature and that can be employed to design active metamaterials. These materials can be categorized as transparent conductive oxides (TCOs) [67,86-90], superconductors [91,92-96], ferroelectrics [such as strontium titanate (STO) and $\mathrm{BaSrTiO}_{3}$ (BST)] $[69,85]$, ferrites and magnetically tunable materials [97-101], liquid crystals (LCs) [102-106], molecules and polymers [107,108], liquid iron $[109,110]$, semiconductors [71], graphene [111-116], and phase-change materials (PCMs) [117-121]. The previous reviews about metamaterial light absorbers have mainly focused on the investigation of theoretical backgrounds, different designs, and applications of passive structures [122-127]. Besides, it has been

(a)

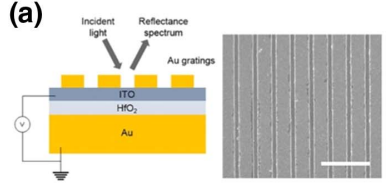

(e)
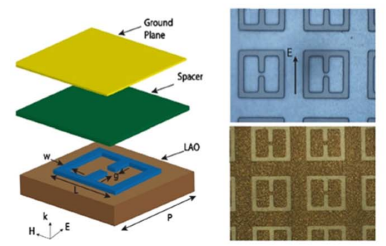

(b)

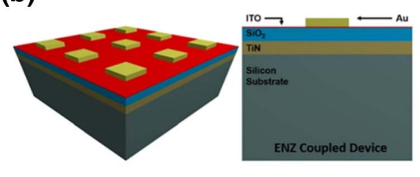

(f)

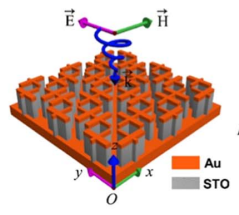

proved that the transmission line theory is also a powerful tool for the investigation of thin absorbing structures [128-131]. However, up to now, a review for the investigation of active MPAs has not been reported. Therefore, as a complementary research study of those available in the literature, in accordance with the MPA designs provided in Fig. 1, in this review paper we examine the different types of MPAs with active characteristics based on the various active materials that can be employed in the designs.

\section{ACTIVE MPAS BASED ON TCOS, SUPERCONDUCTORS, FERROELECTRICS, AND FERRITES}

Indium oxide, cadmium oxide, and zinc oxide are oxide semiconductors capable of being heavily doped to act as films with conducting features. Due to their large band gap, they are transparent in the visible range and thus are known as transparent conducting oxides (TCOs). These materials have been widely used in photovoltaics, organic light-emitting diodes (OLEDs), displays, and electro-optics devices in general $[85,132]$. Since TCOs can be heavily doped, they exhibit high DC conductivity and consequently metal-like optical properties in the nearinfrared (NIR) range. The most popular TCOs are indium tin oxide (ITO) and aluminum-doped $\mathrm{ZnO}$ (AZO) owing to their NIR epsilon-near-zero (ENZ) and plasmonic responses of their highly doped versions. More importantly, the optical properties of ITO and AZO can be tuned in the NIR range by changing the carrier concentration/doping as well as by applying voltage [122-125], which makes these materials noticeable candidates for designing/fabricating tunable metamaterial light absorbers. Shown in Fig. 2(a) is the schematic of an active metamaterial absorber based on ITO [133]. The device operates around the

Fig. 2. Panels (a)-(d) illustrate device schematics of TCO-based NIR MPAs/metamaterials. In panel (a), left figure, an electrically tunable ITO film clamped between a $\mathrm{HfO}_{2}$-coated Au substrate and an array of Au strips [133]. The right figure in this panel shows a SEM image of the MPA. In panel (b), an $\mathrm{Au} / \mathrm{SiO}_{2} / \mathrm{TiN}$ MIM MPA is shown in which a thin film of ITO is implemented for broadening the absorption bandwidth of the MPA and can also be used for tuning purposes [134]. In (c) the schematic and SEM images of an AZO-based metamaterial are presented [135]. Using the effective medium approach, this system can be assumed as a uniaxially anisotropic metamaterial. A GZO-based MIM metamaterial is illustrated in panel (d) [136]. (e) shows the schematic of (left), the mask used for (right top), and the fabricated (right bottom) THz superconductor-based active MPA [137]. The blue SRR shows the YBCO superconductor. (f) Schematic of the THz STO-based MPA [138]. (g) Schematic and SEM image of the THz BST-based metamaterial [139]. (h) schematically shows the microwave ferrite-based MPA [140]. (a) Reprinted from Ref. [133]. Copyright 2015 Springer Nature. (b) Reprinted with permission from Ref. [134]. Copyright 2018 American Chemical Society. (c) Reprinted with permission from Ref. [135]. Copyright 2018 American Chemical Society. (d) Reprinted with permission from Ref. [136]. Copyright 2018 American Chemical Society. (e) Reprinted with permission from Ref. [137]. Copyright 2016 Optical Society of America. (f) Reprinted with permission from Ref. [138]. Copyright 2018 Institute of Physics. (g) Reprinted with permission from Ref. [139]. Copyright 2014 American Institute of Physics. (h) Reprinted with permission from Ref. [140]. Copyright 2016 Elsevier. 
ENZ wavelength of ITO, i.e., $3.7 \mu \mathrm{m}$. As schematically shown in this figure, the active metamaterial absorber is composed of an electrically tunable ITO film clamped between a $\mathrm{HfO}_{2}-$ coated Au substrate and an array of Au strips. With positive and negative electric biases, the carrier concentration of ITO can be decreased (depletion) or increased (accumulation), leading to changes in the optical properties of the ITO film and thus the absorption of the metamaterial. Moreover, it has been recently shown that [schematic depicted in Fig. 2(b)] by encapsulating a thin film of ITO between the top patterned layer and the bottom reflector in an MIM metamaterial absorber, it is possible to tunably broaden the absorption bandwidth of the metamaterial around $1.5 \mu \mathrm{m}$ [134]. The physics behind this broadness is due to the coupling of the epsilon-near-zero mode to the gap plasmon mode supported by the metamaterial. As shown in Fig. 2(c), another example of an active TCO-based structure is a nanopillar-based metamaterial composed of Ge and Al-doped $\mathrm{ZnO}$ (AZO), whose optical properties can be modulated by gating and whose fabrication process is compatible with complementary metal-oxide-semiconductor technology [135]. It was experimentally observed that the NIR resonant reflective response of the metamaterial shifts up to $240 \mathrm{~nm}$ by modifying the gate biases from -4 to $4 \mathrm{~V}$. Adding a bottom reflector to the structure and appropriately picking the spacer layer make the AZO-based metamaterial a practical choice to achieve active absorbers. Instead of having gate-tunable characteristics, galliumdoped zinc oxide (Ga: $\mathrm{ZnO}$ ), as another family member of TCOs, shows tunable features around its ENZ wavelength of around $1.15 \mu \mathrm{m}$ [136]. Panel (d) of Fig. 2 illustrates a schematic of a GZO embedded MIM nanocavity of a total thickness of $230 \mathrm{~nm}$. The cavity, composed of two $24 \mathrm{~nm} \mathrm{Ag}$ top and bottom Ag films, is separated by a passive alumina layer with a thickness of $230 \mathrm{~nm}$. As the optically active component, a $70 \mathrm{~nm}$ thick GZO medium is embedded into the spacer region. Thanks to the presence of GZO, this device shows tunable resonant transmission responses in the NIR region. By turning the bottom Ag layer to a reflective one, it is possible to achieve a metamaterial absorber with an active feature.

Superconductors are another type of active materials whose complex optical conductivity is sensitive to both temperature and electromagnetic fields. This feature gives the advantage of the use of these materials as active components in the metamaterials such that their optical responses can be dynamically tuned via temperature, magnetic field, or electric current [141-143]. Figure 2(e), right panel, shows a unit cell schematic of a reflecting terahertz $(\mathrm{THz})$ perfect absorber that includes a $\mathrm{YBa}_{2} \mathrm{Cu}_{3} \mathrm{O}_{7}$ (YBCO) superconducting film with thickness of $100 \mathrm{~nm}\left[T_{c} \approx 75 \mathrm{~K}\right.$, substrate $\left.\mathrm{LaAlO}_{3}(\mathrm{LAO})\right]$ that is separated from a gold ground plane by a polyimide spacer layer [137]. Photos of the mask (right top panel) used for etching and the resulting YBCO split-ring resonators (SRRs) (right bottom panel) are also provided in Fig. 2(e) as an illustrative example of an active $\mathrm{THz}$ superconductor-based MPA.

Ferroelectric materials are another category of active materials whose dielectric function in the paraelectric phase can be electrically and thermally tuned $[69,85]$. This approach can also be considered as an effective methodology for the realization of active metamaterial absorbers [138,139,144,145]. Strontium titanate (STO) is one of the ferroelectric materials that show temperature-tunable optical responses $[144,138]$. Figure 2(f) illustrates a barium strontium titanate (BST)based active metamaterial light absorber that operates at $\mathrm{THz}$ frequencies with resonant features [138]. It is shown that the resonant absorption peaks of the MIM metamaterial — that is composed of a periodic metal-dielectric stack array placed on a metallic reflector-experience blueshift by increasing the temperature. BST is another commonly used ferroelectric material that exhibits a strong response to an external DC electric field, temperature, and stress [139,145]. A BST-based metamaterial with actively tunable transmission features is shown in Fig. 2(g) [139]. The metamaterial is composed of $\mathrm{Au}$ split ring resonator arrays on a $500 \mathrm{~nm}$ film of BST on an alumina substrate. It is shown once the applied DC electric field is modified from $0 \mathrm{kV} / \mathrm{cm}$ to $13 \mathrm{kV} / \mathrm{cm}, 27 \mathrm{kV} / \mathrm{cm}$, and $33 \mathrm{kV} / \mathrm{cm}$, and the transmission spectra of the metamaterial is noticeably tunable. It is reported that, by introducing a bottom reflector, this design and similar ones for which the top patterned layer is based on BST are capable of exhibiting active absorption characteristics [145].

Ferrites are another type of active materials that present a magnetically tunable response $[97,98]$. These materials are capable of supporting ferromagnetic resonances (FMRs) due to the interaction with a magnetic field of an applied electromagnetic field. Moreover, once an adjustable magnetic field is applied to these materials, the FMR frequency can be tuned. Following this mechanism, ferrite-based metamaterial absorbers with magnetically tunable features have been designed and fabricated [146-148]. Moreover, as an alternative approach for obtaining magnetically tunable metamaterial absorbers, it is also possible to use the ferrite material as the spacer layer of the MIM structure [149,140]. As an example, Fig. 2(h) illustrates a schematic of such a magnetically active metamaterial absorber of this type that operates at microwave frequencies [140].

\section{ACTIVE MPAS BASED ON LCS, POLYMERS, MOLECULES, AND SEMICONDUCTORS}

Liquid crystals are a promising candidate for the development of active photonics due to their large birefringence, low driving threshold, and versatile driving methods. LCs have several phases such as nematic, smectic, cholesteric, and isotropic. These phases can be tuned by external stimuli (e.g., gate voltage and heating) [102-106] and can be employed to achieve active MPAs [150-153]. As an example of the phase transformation, we can refer to nematic LCs. Nematic LCs have a uniform alignment of the director and exhibit uniaxial optical symmetry with two principal refractive indices, i.e., ordinary and extraordinary refractive indices [106]. The applied stimuli change the orientation of the director and therefore cause changes in the refractive index of the LC. This feature makes LCs a versatile candidate to be employed in manufacturing reconfigurable photonic devices. Figure 3(a), left panel, is a schematic depiction of a gate-tunable LC-based metamaterial light absorber that operates around $2.62 \mathrm{THz}$ [150]. As highlighted in the schematics, applying the electric bias leads to a considerable change in the alignment of the LC directors, tuning its refractive index. In this way, it is possible to considerably tune the strength of the absorption peak at $2.62 \mathrm{THz}$. Figure 3(a), right 
(a)
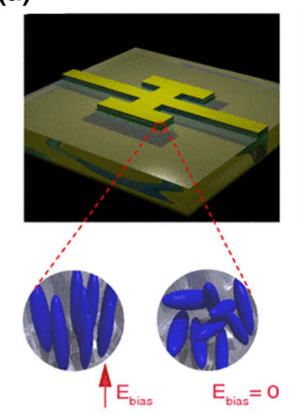

(b)

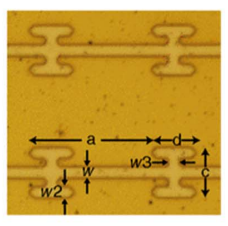

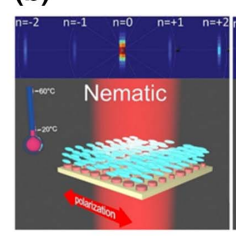

(c)

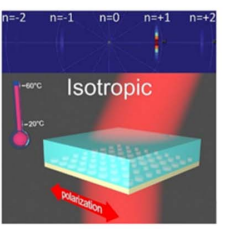

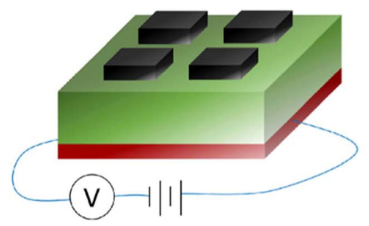

(d)

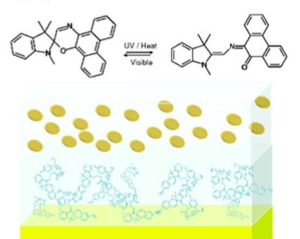

(e)
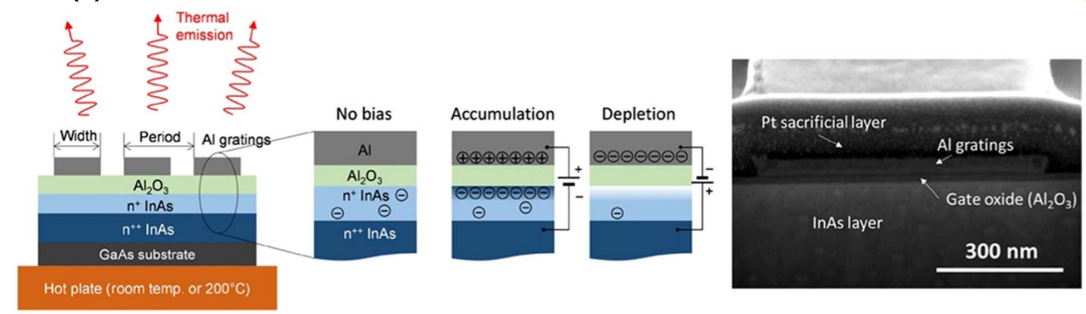

(f)

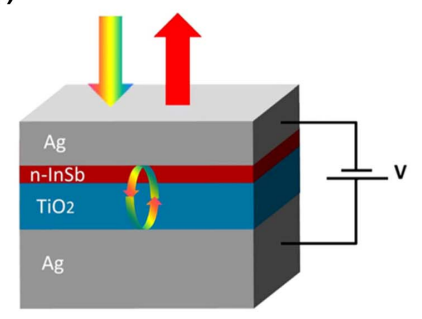

(g)

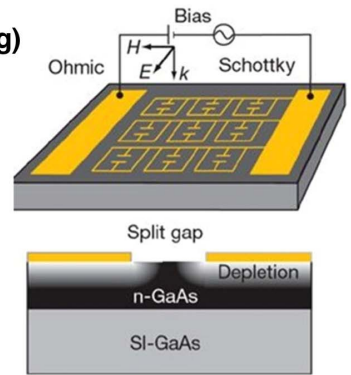

(h)

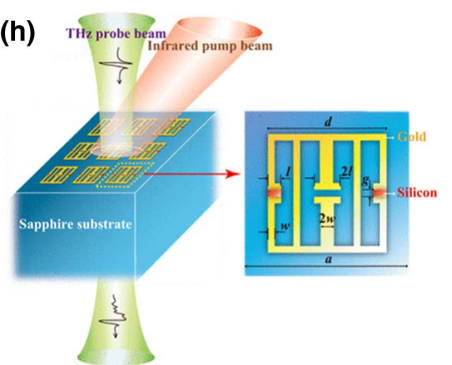

Fig. 3. Schematics of active MPAs and metamaterials based on LCs, polymers, molecules, and semiconductors. (a) shows a rendering of a single unit cell of the THz LC-based MPA (right) and optical microscope image of a portion of the metamaterial array (right). The random alignment of liquid crystal in the unbiased case and for an applied AC bias is presented [150]. Panel (b) illustrates the visible-NIR LC-based metasurface deflection beam switcher for nematic (left) and isotropic (right) states. The nematic and isotropic states are thermally interchanged [153]. A microwave PCO polymer-based MPA that is thermally tunable by Joule heating is presented in panel (c) [154]. (d) shows a visible-NIR photo-driven moleculebased active MPA [155]. (e) [156], (f) [157], and (g) [158] are illustrations of electrically tunable semiconductor-based MPAs and metamaterials that operate within the MIR, visible-NIR, and THz ranges, respectively. Panel (h) is an illustration of an optically tunable THz semiconductor-based metamaterial [159] that can also be employed for the realization of dynamic MPAs [160]. (a) Reprinted with permission from Ref. [150]. Copyright 2013 American Physical Society. (b) Reprinted with permission from Ref. [153]. Copyright 2018 American Chemical Society. (c) Reprinted with permission from Ref. [154]. Copyright 2018 American Chemical Society. (d) Reprinted with permission from Ref. [155]. Copyright 2014 John Wiley and Sons. (e) Reprinted from Ref. [156]. (C) The Authors, some rights reserved; exclusive licensee American Association for the Advancement of Science. Distributed under a Creative Commons Attribution NonCommercial License 4.0 (CC BY-NC) http://creativecommons.org/licenses/bync/4.0/. (f) Reprinted from Ref. [157]. Copyright 2018 Springer Nature. (g) Reprinted with permission from Ref. [158]. Copyright 2006 Springer Nature. (h) Reprinted with permission from Ref. [159]. Copyright 2011 American Physical Society.

panel, shows optical microscope image of a portion of the metamaterial array where the unit cell has the dimensions of $a=50$, $c=20, d=16, w=w_{2}=4: 5$, and $w_{3}=5 \mu \mathrm{m}$. Figure 3(b) presents visible-NIR temperature-tunable beam steering of a dielectric metasurface by modifying the liquid crystal state from nematic to isotropic [153]. Based on a similar mechanism, it is also possible to design and fabricate a temperature-tunable LC-based active metamaterial absorber.

Responsive polymers are another type of materials with tunable characteristics that can consequently be employed as building block components in active MPAs. Responsive polymers that show an active response to heat, light, electricity, or chemicals can be cut to smaller chains [107]. This attribute enables the switching of polymer properties with external stimuli. Responsive polymers can also be achieved by incorporating molecular switches (e.g., azobenzene and spiropyran) [108]. A novel polymer-based light absorber, which is made of polycyclooctene (PCO) polymer composites and multi-walled carbon nanotubes (MWCNTs), that acts as a frequencyselective surface is illustrated in Fig. 3(c) [154]. This absorber is capable of achieving wide frequency-tunable light absorption from 10 to $17.2 \mathrm{GHz}$ by actively adjusting the sheet resistance of the highly conductive ground plane under DC voltages up to $22 \mathrm{~V}$. Photoswitchable polymer films are another example of polymers with light-tunable characteristic. Figure 3(d) shows the schematics of an optically driven super-absorbing system that operates in the visible-NIR regions [155]. This absorber is realized in a stack composed of a thin polymer-based photoswitchable film as the spacer, which is embedded between a metal mirror and top film composed of gold nanoparticles. In such a system, the reflectivity of the metal mirror could be dropped down to a few percent or recovered to more than 95\% dynamically by UV or visible illumination, respectively.

As the key materials for the fabrication of electronics and photonics devices, semiconductors have the capability of having tunable electronic and optical responses. Recently, there have been 
numerous reports on using optically [71,159,160-162] and electrically tunable [156-158] semiconductors in obtaining active metamaterials and active PMAs. Figure 3(e) shows a schematic of a InAs-based plasmonic metasurface that acts as an active thermal emitter within 4 to $10 \mu \mathrm{m}$. Note that, according to Kirchhoff's law, the spectral-directional emissivity of the structure is the same as its spectral-directional absorptivity. In this structure, a low-doped InAs layer that acts as the voltage-tunable active medium is embedded inside the MIM-like cavity and is responsible for the tunable thermal emission of the system. A cross-sectional scanning electron microscope (SEM) image of the active metasurface is also provided on the right-hand side of panel (e) [156]. As another example, Fig. 3(f) shows how an ultrathin n-type doped indium antimonide integrated into a subwavelength thick optical nanocavity can result in an electrically tunable perfect light absorber in the visible and near infrared ranges [157]. This structure may also be used as the optical modulators in the NIR. A schematic of an active THz metamaterial device is depicted in panel (g) of Fig. 3 [158]. This active metamaterial device-which works in transmission mode and consists of an array of gold electric resonators fabricated on n-type GaAs as the voltage-controlled active medium-is capable of efficient real-time control and manipulation of $\mathrm{THz}$ radiation. The metamaterial array and substrate together effectively form a Schottky diode, which enables the modulation of $\mathrm{THz}$ transmission by 50 percent, an order of magnitude improvement over existing devices. By introducing a bottom reflector layer beneath the semiconductor medium and gating the structure appropriately, an active THz MPA can be achieved. It is also possible to control the optical properties of a photoconductive semiconductor, e.g., Si, by light. Figure 3(h) shows perspective and top view schematics of an active $\mathrm{THz}$ Si-based metamaterial that presents optically implemented blueshift in its transmission response [159]. Obviously, adding a bottom reflector with an appropriate spacing layer makes this design an active MPA [163].

\section{ACTIVE MPAS BASED ON GRAPHENE AND PCMS}

After the rise of graphene, the two-dimensional periodic array of carbon atoms arranged in a honeycomb lattice [1,2], a great deal of attention has been attracted to its potential applications in optoelectronics [3] and plasmonics [4]. The surface conductivity of graphene $\left(\sigma_{g}\right)$ can be effectively modulated via tuning of chemical potential $(\mu)$ through chemical doping, electrostatic/magnetostatic gating, optical pumping, and temperature [1,2,164-166]. Depending on the intensity of the incident light, graphene may also show nonlinear responses, giving the opportunity of obtaining active graphene-based metamaterials by optical pumping. In this case, $\sigma_{g}$ would depend on light intensity [167]. When $\operatorname{Im}\left(\sigma_{g}\right)>0$, graphene behaves like a very thin metal layer capable of supporting transverse-magnetic (TM) guided plasmonic modes [163,168-172] within the infrared and $\mathrm{THz}$ ranges. Therefore, if they are designed appropriately, it is possible to achieve active graphenebased MPAs that operate in a broad range of frequency with tunable (electrically, magnetically, optically, and thermally) features (see, e.g., [173-177,178,179]). Figure 4(a) shows a widely tunable mid-infrared (MIR) metasurface composed of optical antennas on an unpatterned graphene sheet that is incorporated into a subwavelength-thick optical cavity to create an electrically tunable MPA [173]. By switching the absorber in and out of the critical coupling condition via the applied gate voltage on graphene, a modulation depth of up to $100 \%$ can be achieved. In particular, an ultrathin optical modulator (thickness $<\lambda_{0} / 10$ ) with high speed (up to $20 \mathrm{GHz}$ ) over a broad wavelength range $(5-7 \mu \mathrm{m})$ is obtained by this structure. Figure 4(b) exhibits an MIR metamaterial composed of graphene disks that is capable of exhibiting light absorption. It is shown that by nanopatterning a graphene layer into an array of closely packed graphene nanodisks, its absorption efficiency can be increased from less than 3\% to 30\% in the infrared region of the spectrum. Moreover, by incorporating graphene nanodisk (nanodot) arrays into an active device (covered by ion gel and gated by gold), it is demonstrated that this enhanced absorption efficiency is voltage tunable [174]. As mentioned earlier, by introducing a bottom reflector in this structure and taking a spacer appropriately, it is possible to increase light absorption from $30 \%$ to $>90 \%$ and achieve active MIR graphene-based MPAs $[175,176]$. By patterning graphene as anti-dot arrays and employing the patterned structure in a cavity, like the one shown in Fig. 4(c), it is also possible to effectively tune the MIR nearly perfect resonant absorption responses of the structure [177]. This figure schematically presents the integration of the graphene antidote MPA with a patterned hexagonal boron nitride $(\mathrm{hBN})$ as a polar material. This integration and thus hybridization of graphene plasmons and hBN phonons $[187,180]$ leads to the appearance of multi plasmonic-phononic resonances and thus the improvement of the active MPA's characteristics. It is also possible to achieve a MPA using unpatterned graphene layer(s). Figure 4(d) illustrate a hybrid light absorber composed of graphene and a 1D photonic crystal. Due to the support of Tamm SPPs, the THztunable resonant absorption characteristic is observed in this structure [178]. A multilayer graphene-dielectric structure is shown in Fig. 4(e). This structure, which may behave as a hyperbolic metamaterial within the MIR, far-infrared (FIR), and $\mathrm{THz}$ ranges, depending on the thickness and material of the dielectric layers - is also capable of acting as an active MPA [179]. Zero-index photonic crystals have also found practical applications [188]. Combining these structures with lossy and tunable elements can also provide tunable absorption characteristics with broadened features.

Phase-change materials, such as vanadium dioxide $\left(\mathrm{VO}_{2}\right)$ and $\mathrm{Ge}_{3} \mathrm{Sb}_{2} \mathrm{Te}_{6}$ (GST), belong to another category of active materials and exhibit reversible insulator-to-metal transitions (IMT) $[124,125]$. This reversible optical response is observed in PCMs' amorphous and crystalline states and can be obtained by external stimuli such as heat, light, and voltage. The mentioned phase transitions lead to dramatic change in PCMs' complex refractive indices. Consequently, this attribute makes PCMs promising candidates to be used as the building block components in active metamaterials such as light absorbers (see, e.g., [181-185]), switches, and modulators [120,121]. Figure 4(f) schematically illustrates hybrid gold- $\mathrm{VO}_{2}$ designs for which the grating (left panel) and cylindrical patches (right panel) are made of gold [181]. Using these designs, two 
(a)
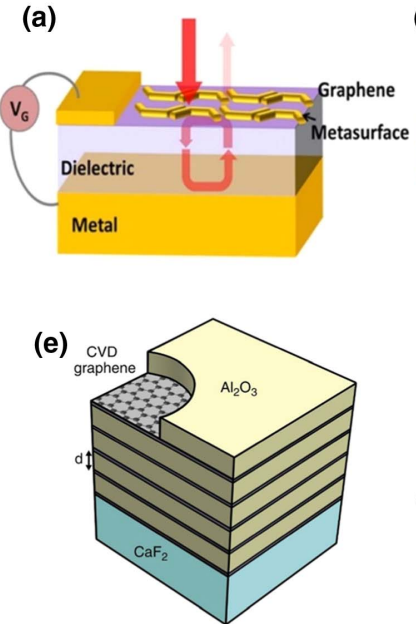

(h)

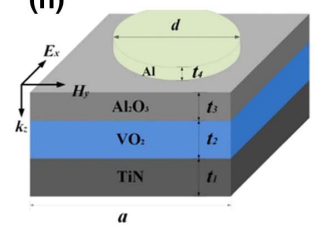

(i) (b)
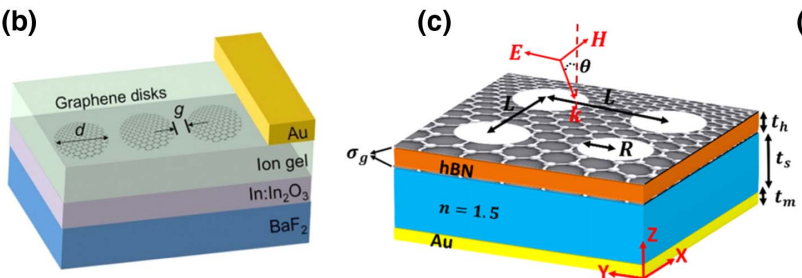

(d)

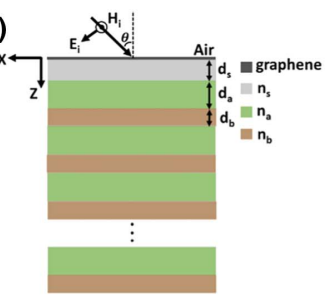

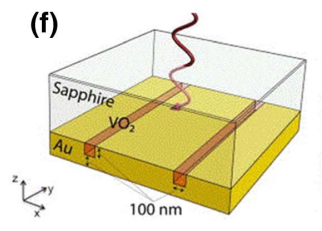
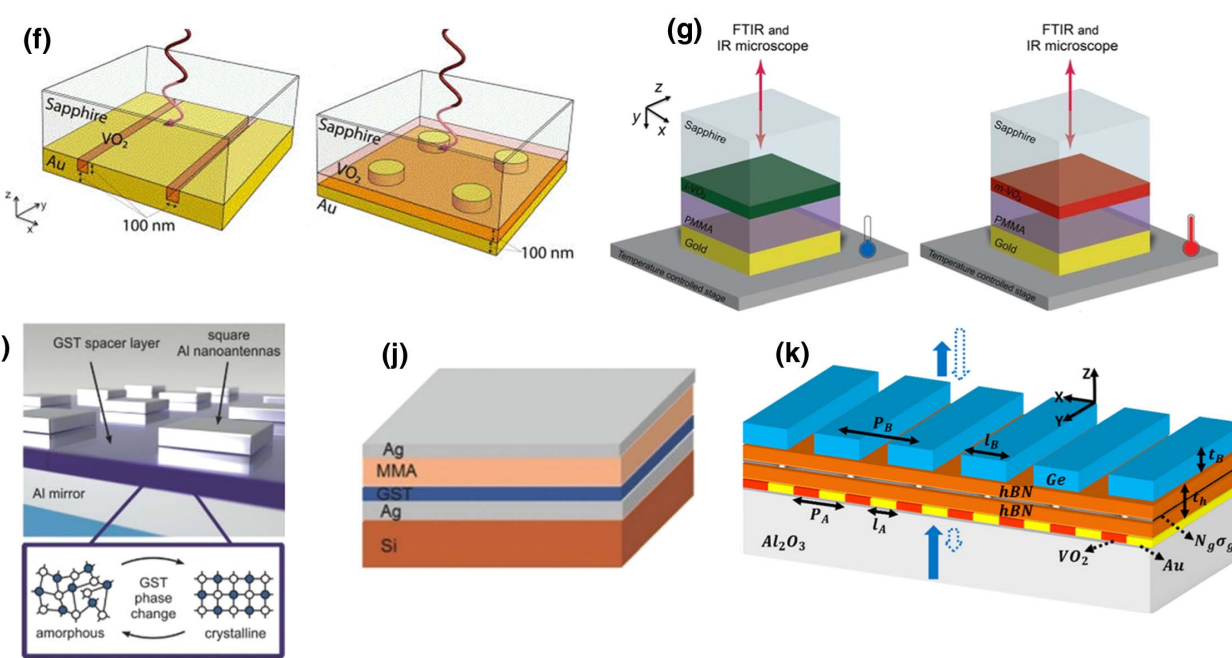

(j)
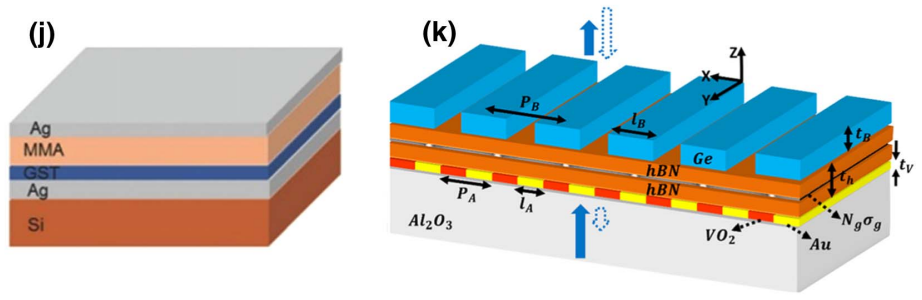

Fig. 4. Schematics of active MPAs and structures based on graphene and PCMs. (a) An MIR graphene-based MPA composed of a gold metasurface on graphene, dielectric spacer (AlOx), and a reflector (Al) [173]. (b) An active MIR metamaterial absorber based on graphene disks [174]. In panel (c) an MIR graphene-based MPA is illustrated in which the top graphene-hBN-graphene layers are patterned as an anti-dot structure [177]. (d) illustrates a graphene-based 1D PC that acts as a THz narrowband perfect absorber [178]. (e) A multilayer graphene-dielectric metamaterial that can also be employed as an active MPA in the IR and THz regions [115,163,179,180]. (f) shows an IR MPA based on patterned $\mathrm{VO}_{2}$ films [181], while in (g) [182] and (h) [183] active IR MPAs are achieved using unpatterned films of $\mathrm{VO}_{2}$. GST-based MPAs employed in patterned [184] and unpatterned [185] visible-NIR MIM structures are shown in panels (i) and (j), respectively. (k) illustrates a schematic of the $\mathrm{VO}_{2}$-hBN-graphenebased bi-functional metamaterial for bi-tunable asymmetric transmission and nearly perfect resonant absorption characteristics [186]. (a) Reprinted with permission from Ref. [173]. Copyright 2014 American Chemical Society. (b) Reprinted with permission from Ref. [174]. Copyright 2014 American Chemical Society. (c) Reprinted with permission from Ref. [177]. Copyright 2018 Optical Society of America. (d) Reprinted with permission from Ref. [178]. Copyright 2017 Optical Society of America. (e) Reprinted from Ref. [179]. Copyright 2016 Springer Nature. (f) Reprinted with permission from Ref. [181]. Copyright 2015 American Institute of Physics. (g) Reprinted from Ref. [182]. Copyright 2015 Springer Nature. (h) Reprinted with permission from Ref. [183]. Copyright 2017 Optical Society of America. (i) Reprinted with permission from Ref. [184]. Copyright 2015 John Wiley and Sons. (j) Reprinted with permission from Ref. [185]. Copyright 2018 John Wiley and Sons. (k) Reprinted with permission from Ref. [186]. Copyright 2019 Optical Society of America.

thermally tunable infrared resonant MPAs are achieved. It is observed that the absorption intensity is tuned from $90 \%$ to $20 \%$ and $96 \%$ to $32 \%$ using hybrid gold $-\mathrm{VO}_{2}$ grating and cylindrical patches, respectively, by heating up the absorbers above the phase transition temperature of $\mathrm{VO}_{2}$ (i.e., $68^{\circ} \mathrm{C}$ ). It is also possible to obtain broadband nearly perfect absorption by unpatterned MIM structures. Figure 4(g) illustrates an IR active MPA that is composed of a gold bottom reflector, a PMMA layer as the spacer, and an ultrathin $20 \mathrm{~nm}$ film of $\mathrm{VO}_{2}$ at the top. By changing temperature and thus transitioning the phase of $\mathrm{VO}_{2}$ from insulator to metal, it is possible to switch absorption from $\sim 15 \%$ to $\sim 90 \%$ and vice versa [182]. We can use $\mathrm{VO}_{2}$ as the spacer in the MIM structure to amend the cavity length and therefore actively tune the absorption response of the structure. Figure $4(\mathrm{~h})$ provides an example in this regard [183]. As shown in this schematic, TiN is considered to be the reflecting layer, $\mathrm{Al}$ disks are chosen as the top patterned medium, and $\mathrm{Al}_{2} \mathrm{O}_{3}$ and $\mathrm{VO}_{2}$ jointly form the spacer media in this MIM cavity. By the phase transmission of $\mathrm{VO}_{2}$, the cavity length is modified, and thus the absorption response of the metamaterial is actively tuned. Figure 4(i) shows a schematic of a thermally active GST-based MPA with multispectral thermal imaging capability that operates within the $3-5 \mu \mathrm{m}$ range [184]. The basic building blocks of the design are absorber pixels consisting of an array of square $\mathrm{Al}$ nanoantennas stacked above a GST spacer layer and an $\mathrm{Al}$ mirror. As depicted in Fig. 4(j), it is also possible to use GST as a cavity length modifier in a lithography-free asymmetric MIM structure. In this design, the spacer is composed of GST and methyl methacrylate layers and is bounded with top $(18 \mathrm{~nm})$ and bottom $(50 \mathrm{~nm}) \mathrm{Ag}$ films. This structure, which operates in the visible and NIR ranges, shows multi-narrowband perfect absorption with a maximum of $99.8 \%$ at a specific incident angle and polarization state when the GST is in the amorphous phase. The absorption bands blueshift and broaden after switching to the crystalline phase [185]. It is noteworthy that the 
potential application of PCMs is not limited to the visible and IR regions; there are also some reports on taking advantage of PCMs' active optical responses in the microwave and $\mathrm{THz}$ regions $[119,120]$. Moreover, very recently, it has been theoretically reported that by combining $\mathrm{VO}_{2}, \mathrm{hBN}$, and graphene in one structure it is possible to obtain a bi-tunable metamaterial with bi-functional MPA and asymmetric light transmitter characteristics in the MIR region [186], see Fig. 4(k).

\section{ACTIVE MPAS BASED ON MEMS, MICROFLUIDIC CHANNELS, AND ELASTIC SUBSTRATES}

In the above-mentioned examples, the metamaterials' tuning was demonstrated via modification of the optical properties of their building block components. The alternative way to achieve tunability is to change the physical distance between the metamaterial elements. This leads to a new tool for the optimization of the resonant responses of the system and thus provides tunability. This aim is possible by using MEMS/ NEMS - in which the electrical and mechanical elements are integrated - and also microfluidic channels and stretchable materials in the system [75-83,189-195]. MEMS/NEMS are composed of micro- or nanometer scale components and have the ability to function in the micro- and nano-scale. Therefore, by fabricating metamaterials as MEMS/NEMS, we may achieve mechanically actuated tuning. Figure 5(a) schematically presents a MEMS-based tunable MPA that operates at $\mathrm{THz}$ frequencies [190]. The basic idea behind employing MEMS in this device is the strong sensitivity of the metamaterials' electromagnetic response to position. In other words, in this structure, meta-atoms that support strongly localized modes are combined with suspended flat membranes that can be driven electrostatically. As mentioned above, integrating metamaterials with microfluidic channels-i.e., the field of metafluidic metamaterials [194] — has been also considered as one of the effective ways to achieve tunable devices that are beneficial for applications such as MPAs and sensors. Figure 5(b) shown a photograph of a switchable X-band MPA that works based on injecting eutectic gallium-indium (EGaIn) liquid metal alloy using microfluidic channels [194]. The switchable response is observed once the optical responses of the device when the microfluidic channels are empty compared to the case for which they are filled with the liquid metal. Using elastic substrates is also another way of achieving flexible and reconfigurable MPAs. Photo of a planar flexible MPA that operates at around $2 \mathrm{GHz}$ is shown in Fig. 5(c) [195]. Bending the flexible metamaterial and thereby modifying the geometrical parameters leads to observation of the tuning of the resonant absorption peaks of the structure.

\section{CONCLUSION AND PERSPECTIVE}

In the present review paper, we first outlined the different types of nearly perfect metamaterial light absorbers with narrowband and broadband responses. Then, through reviewing different types of active materials (i.e., TCOs, superconductors, ferroelectrics, ferrites, LCs, polymers, molecules, semiconductors, graphene, and PCMs), we provided diverse examples of active MPAs that operate in different frequency ranges [from gigahertz $(\mathrm{GHz})$ to the visible region) and are realized by employing numerous tuning mechanisms, i.e., electrically, optically, magnetically, thermally, and mechanically. Finally, by providing some examples on MPAs based on MEMS, microfluidic channels, and elastic substrates, different mechanisms and technologies of tuning the operational response of the nearly perfect light absorbers have been provided. According to the comprehensive examination we performed in this review paper,

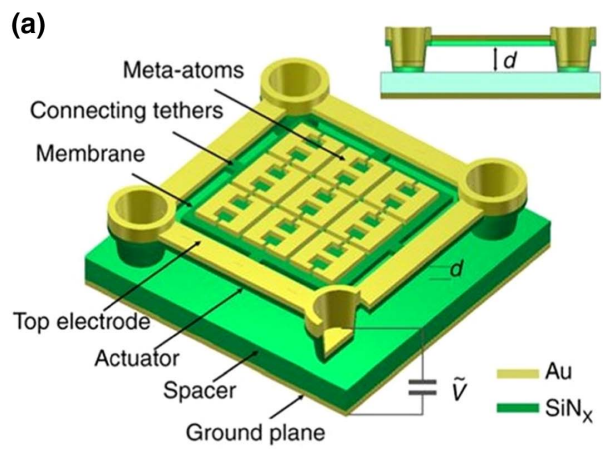

(b)
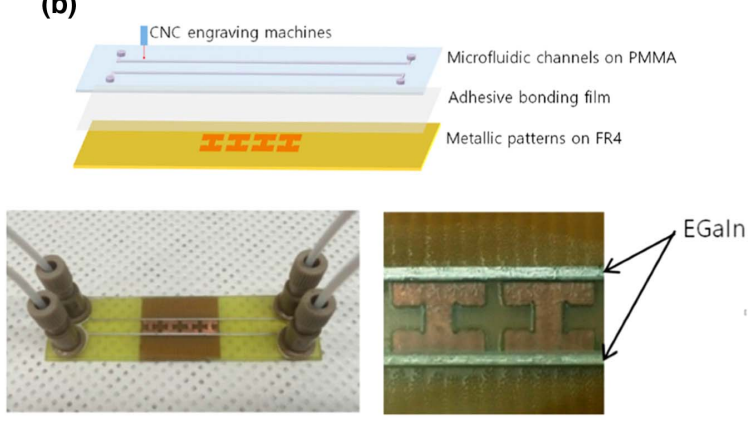

(c)

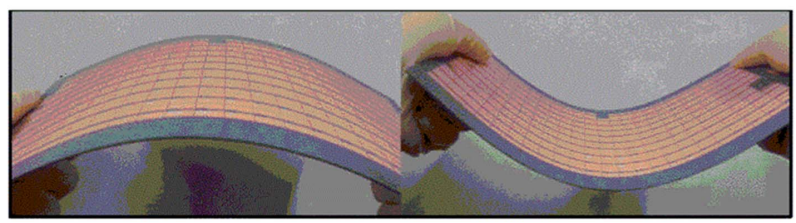

Fig. 5. Schematics/photos of active MPAs based on MEMS, microfluidic channels, and elastic substrates. (a) Unit cell of the THz MEMS-based active MPA. The vertical distance between the meta-atoms and the ground plane can be tuned electrostatically [190]. (b) Schematic and photograph of frequency-switchable X-band MPA that operates based on the injection of eutectic gallium-indium (EGaIn) liquid metal alloy [194]. (c) Photos of the flexible and elastic microwave MPA [195]. (a) Reprinted from Ref. [190]. Copyright 2017 Springer Nature. (b) Reprinted with permission from Ref. [194]. Copyright 2015 MPDI. (c) Reprinted with permission from Ref. [195]. Copyright 2014 American Chemical Society. 
different types of active materials and tuning mechanisms could be selected to achieve appropriate active MPAs based on the device structure, operation wavelength, and the application scenario. As has been investigated, numerous studies and papers in the field of active metamaterial light absorbers have been performed and published, but there is still considerable space to work on and develop active MPAs based on the materials and mechanisms investigated in this review paper. There is considerable potential to design and fabricate tunable metamaterial absorbers, and more progress in this field will pave the way for the realization of active MPAs that can be employed in devices such as tunable thermal emitters, sensors, filters, photodetectors, and solar cells.

Funding. DPT-HAMIT and Türkiye Bilimsel ve Teknolojik Araştirma Kurumu (TÜBITAK) (113E331, 114E374, 115F560).

Acknowledgment. The authors acknowledge support from DPT-HAMIT and TÜBITAK. E. O. acknowledges support from the Turkish Academy of Sciences (TUBA).

\section{REFERENCES}

1. V. G. Veselago, "The electrodynamics of substances with simultaneously negative values of $\varepsilon$ and $\mu$," Sov. Phys. Usp. 10, 509-514 (1968).

2. A. Boardman, "Pioneers in metamaterials: John Pendry and Victor Veselago," J. Opt. 13, 020401 (2011).

3. D. R. Smith, W. J. Padilla, D. Vier, S. C. Nemat-Nasser, and S. Schultz, "Composite medium with simultaneously negative permeability and permittivity," Phys. Rev. Lett. 84, 4184-4187 (2000).

4. H. Chen, B.-I. Wu, B. Zhang, and J. A. Kong, "Electromagnetic wave interactions with a metamaterial cloak," Phys. Rev. Lett. 99, 063903 (2007).

5. N. Seddon and T. Bearpark, "Observation of the inverse Doppler effect," Science 302, 1537-1540 (2003).

6. A. Fang, T. Koschny, and C. M. Soukoulis, "Lasing in metamaterial nanostructures," J. Opt. 12, 024013 (2010).

7. Z. Li, M. Mutlu, and E. Ozbay, "Chiral metamaterials: from optical activity and negative refractive index to asymmetric transmission," J. Opt. 15, 023001 (2013).

8. M. Lapine, I. V. Shadrivov, D. A. Powell, and Y. S. Kivshar, "Magnetoelastic metamaterials," Nat. Mater. 11, 30-33 (2012).

9. N. I. Landy, S. Sajuyigbe, J. Mock, D. Smith, and W. Padilla, "Perfect metamaterial absorber," Phys. Rev. Lett. 100, 207402 (2008).

10. N. Mattiucci, M. J. Bloemer, N. Aközbek, and G. D'Aguanno, "Impedance matched thin metamaterials make metals absorbing," Sci. Rep. 3, 3203 (2013).

11. H. Deng, Z. Li, L. Stan, D. Rosenmann, D. Czaplewski, J. Gao, and $X$. Yang, "Broadband perfect absorber based on one ultrathin layer of refractory metal," Opt. Lett. 40, 2592-2595 (2015).

12. K. B. Alici, F. Bilotti, L. Vegni, and E. Ozbay, "Experimental verification of metamaterial based subwavelength microwave absorbers," J. Appl. Phys. 108, 083113 (2010).

13. S. Bhattacharyya and K. Vaibhav Srivastava, "Triple band polarization-independent ultra-thin metamaterial absorber using ELC resonator," J. Appl. Phys. 115, 064508 (2014).

14. H. Li, L. Hua Yuan, B. Zhou, X. P. Shen, Q. Cheng, and T. J. Cui, "Ultrathin multiband gigahertz metamaterial absorbers," J. Appl. Phys. 110, 014909 (2011).

15. S. Bhattacharyya, S. Ghosh, and K. V. Srivastava, "Triple band polarization-independent metamaterial absorber with bandwidth enhancement at X-band," J. Appl. Phys. 114, 094514 (2013).

16. J. W. Park, P. V. Tuong, J. Y. Rhee, K. W. Kim, W. H. Jang, E. H. Choi, L. Y. Chen, and Y. Lee, "Multi-band metamaterial absorber based on the arrangement of donut-type resonators," Opt. Express 21, 9691-9702 (2013).
17. X. Shen, T. J. Cui, J. Zhao, H. F. Ma, W. X. Jiang, and H. Li, "Polarizationindependent wide-angle triple-band metamaterial absorber," Opt. Express 19, 9401-9407 (2011).

18. P. Munaga, S. Ghosh, S. Bhattacharyya, and K. V. Srivastava, "A fractal based compact broadband polarization insensitive metamaterial absorber using lumped resistors," Microwave and Opt. Technol. Lett. 58, 343-347 (2016).

19. Y. Z. Cheng, Y. Wang, Y. Nie, R. Z. Gong, X. Xiong, and X. Wang, "Design, fabrication and measurement of a broadband polarizationinsensitive metamaterial absorber based on lumped elements," J. Appl. Phys. 111, 044902 (2012).

20. M. G. Nielsen, D. K. Gramotnev, A. Pors, O. Albrektsen, and S. I. Bozhevolnyi, "Continuous layer gap plasmon resonators," Opt. Express 19, 19310-19322 (2011).

21. H. T. Chen, "Interference theory of metamaterial perfect absorbers," Opt. Express 20, 7165-7172 (2012).

22. Y. Bai, L. Zhao, D. Ju, Y. Jiang, and L. Liu, "Wide-angle, polarizationindependent and dual-band infrared perfect absorber based on L-shaped metamaterial," Opt. Express 23, 8670-8680 (2015).

23. H. Hajian, A. Ghobadi, B. Butun, and E. Ozbay, "Nearly perfect resonant absorption and coherent thermal emission by hBN-based photonic crystals," Opt. Express 25, 31970-31987 (2017).

24. Z. Yong, S. Zhang, C. Gong, and S. He, "Narrow band perfect absorber for maximum localized magnetic and electric field enhancement and sensing applications," Sci. Rep. 6, 24063 (2016).

25. J. Kim, K. Han, and J. W. Hahn, "Selective dual-band metamaterial perfect absorber for infrared stealth technology," Sci. Rep. 7, 6740 (2017).

26. N. Liu, M. Mesch, T. Weiss, M. Hentschel, and H. Giessen, "Infrared perfect absorber and its application as plasmonic sensor," Nano Lett. 10, 2342-2348 (2010)

27. Z. Li, S. Butun, and K. Aydin, "Large-area, lithography-free super absorbers and color filters at visible frequencies using ultrathin metallic films," ACS Photon. 2, 183-188 (2015).

28. C. Park, V. R. Shrestha, S. Lee, and D. Choi, "Trans-reflective color filters based on a phase compensated etalon enabling adjustable color saturation," Sci. Rep. 6, 25496 (2016).

29. Z. Li, S. Butun, and K. Aydin, "Ultranarrow band absorbers based on surface lattice resonances in nanostructured metal surfaces," ACS Nano 8, 8242-8248 (2014).

30. R. Badugu and J. R. Lakowicz, "Tamm state-coupled emission: effect of probe location and emission wavelength," J. Phys. Chem. C 118, 21558-21571 (2014).

31. J. M. R. Sousa Dias, C. Gong, Z. A. Benson, and M. S. Leite, "Lithography-free, omnidirectional, CMOS-compatible AICu alloys for thin-film superabsorbers," Adv. Opt. Mater. 6, 1700830 (2018).

32. C. Hu, L. Liu, Z. Zhao, X. Chen, and X. Luo, "Mixed plasmons coupling for expanding the bandwidth of near-perfect absorption at visible frequencies," Opt. Express 17, 16745-16749 (2009).

33. K. Aydin, V. E. Ferry, R. M. Briggs, and H. A. Atwater, "Broadband polarization-independent resonant light absorption using ultrathin plasmonic super absorbers," Nat. Commun. 2, 517 (2011).

34. M. Keshavarz Hedayati, M. Javaherirahim, B. Mozooni, R. Abdelaziz, A. Tavassolizadeh, V. Chakravadhanula, V. Zaporojtchenko, T. Strunkus, F. Faupel, and M. Elbahri, "Design of a perfect black absorber at visible frequencies using plasmonic metamaterials," Adv. Mater. 23, 5410-5414 (2011).

35. M. G. Nielsen, A. Pors, O. Albrektsen, and S. I. Bozhevolnyi, "Efficient absorption of visible radiation by gap plasmon resonators," Opt. Express 20, 13311-13319 (2012).

36. A. Ghobadi, H. Hajian, M. Gokbayrak, S. A. Dereshgi, A. Toprak, B. Butun, and E. Ozbay, "Visible light nearly perfect absorber: an optimum unit cell arrangement for near absolute polarization insensitivity," Opt. Express 25, 27624-27634 (2017).

37. A. Ghobadi, S. A. Dereshgi, H. Hajian, B. Bozok, B. Butun, and E. Ozbay, "Ultra-broadband, wide angle absorber utilizing metal insulator multilayers stack with a multi-thickness metal surface texture," Sci. Rep. 7, 4755 (2017).

38. S. A. Dereshgi, A. Ghobadi, H. Hajian, B. Butun, and E. Ozbay, "Ultra-broadband, lithography-free, and large-scale compatible 
perfect absorbers: the optimum choice of metal layers in metal-insulator multilayer stacks," Sci. Rep. 7, 14872 (2017).

39. A. Ghobadi, S. A. Dereshgi, H. Hajian, G. Birant, B. Butun, A. Bek, and E. Ozbay, "97 percent light absorption in an ultrabroadband frequency range utilizing an ultrathin metal layer: randomly oriented, densely packed dielectric nanowires as an excellent light trapping scaffold," Nanoscale 9, 16652-16660 (2017).

40. A. Ghobadi, H. Hajian, A. Rahimi Rashed, B. Butun, and E. Ozbay, "Tuning the metal filling fraction in metal-insulator-metal ultrabroadband perfect absorbers to maximize the absorption bandwidth," Photon. Res. 6, 168-176 (2018).

41. X. Chen, J. H. Wu, C. R. Liu, and P. Cao, "Ultra-broadband wideangle perfect absorber in the visible regime through a thin gratinginsulator-metal structure," J. Opt. Soc. Am. B 36, 153-161 (2019).

42. X. Liu, T. Tyler, T. Starr, A. F. Starr, N. M. Jokerst, and W. J. Padilla, "Taming the blackbody with infrared metamaterials as selective thermal emitters," Phys. Rev. Lett. 107, 045901 (2011).

43. F. Ding, J. Dai, Y. Chen, J. Zhu, Y. Jin, and S. I. Bozhevolnyi, "Broadband near-infrared metamaterial absorbers utilizing highly lossy metals," Sci. Rep. 6, 39445 (2016).

44. Y. Cui, K. H. Fung, J. Xu, H. Ma, Y. Jin, S. He, and N. X. Fang, "Ultrabroadband light absorption by a sawtooth anisotropic metamaterial slab," Nano Lett. 12, 1443-1447 (2012).

45. C. Yang, C. Ji, W. Shen, K. Lee, Y. Zhang, X. Liu, and L. J. Guo, "Compact multilayer film structures for ultrabroadband, omnidirectional, and efficient absorption," ACS Photon. 3, 590-596 (2016).

46. Y. K. Zhong, Y. Lai, M. T. B. Chen, S. M. Fu, P. Yu, and A. Lin, "Omnidirectional, polarization-independent, ultra-broadband metamaterial perfect absorber using field-penetration and reflectedwave-cancellation," Opt. Express 24, A832-A845 (2016).

47. A. Ghobadi, H. Hajian, S. A. Dereshgi, B. Bozok, B. Butun, and E. Ozbay, "Disordered nanohole patterns in metal-insulator multilayer for ultra-broadband light absorption: atomic layer deposition for lithography free highly repeatable large scale multilayer growth," Sci. Rep. 7, 15079 (2017).

48. M. E. Stewart, C. R. Anderton, L. B. Thompson, J. Maria, S. K. Gray, J. A. Rogers, and R. G. Nuzzo, "Nanostructured plasmonic sensors," Chem. Rev. 108, 494-521 (2008).

49. N. I. Landy, C. M. Bingham, T. Tyler, N. Jokerst, D. R. Smith, and W. J. Padilla, "Design, theory, and measurement of a polarization insensitive absorber for terahertz imaging," Phys. Rev. B 79, 125104 (2009).

50. Tittl, A. U. Michel, M. Schaaferling, X. Yin, B. Gholipour, L. Cui, M. Wuttig, T. Taubner, F. Neubrech, and H. A. Giessen, "Switchable mid-infrared plasmonic perfect absorber with multispectral thermal imaging capability," Adv. Mater. 27, 4597-4603 (2015).

51. S. Yokogawa, S. P. Burgos, and H. A. Atwater, "Plasmonic color filters for CMOS image sensor applications," Nano Lett. 12, 43494354 (2012).

52. A. Ghobadi, H. Hajian, M. C. Soydan, B. Butun, and E. Ozbay, "Lithography-free planar band-pass reflective color filter using a series connection of cavities," Sci. Rep. 9, 290 (2019).

53. E. Rephaeli and S. Fan, "Absorber and emitter for solar thermophotovoltaic systems to achieve efficiency exceeding the ShockleyQueisser limit," Opt. Express 17, 15145-15159 (2009).

54. T. J. Liu and J. Takahara, "Ultrabroadband absorber based on single-sized embedded metal-dielectric-metal structures and application of radiative cooling," Opt. Express 25, 1443-1447 (2017).

55. F. P. G. de Arquer, A. Mihi, and G. Konstantatos, "Large-area plasmonic-crystal-hot-electron-based photodetectors," ACS Photon. 2, 950-957 (2015)

56. T. G. U. Ghobadi, A. Ghobadi, E. Ozbay, and F. Karadas, "Strategies for plasmonic hot-electron-driven photoelectrochemical water splitting," Chem. Photo Chem. 2, 1-23 (2018).

57. J. Fang, Q. Liu, W. Zhang, J. Gu, Y. Su, H. Su, C. Guoa, and D. Zhang, "Ag/diatomite for highly efficient solar vapor generation under one-sun irradiation," J. Mater. Chem. A 5, 17817-17821 (2017).

58. A. V. Krasavin and N. I. Zheludev, "Active plasmonics: controlling signals in Au/Ga waveguide using nanoscale structural transformations," Appl. Phys. Lett. 84, 1416-1418 (2004).

59. K. F. MacDonald, Z. L. Sámson, M. I. Stockman, and N. I. Zheludev, "Ultrafast active plasmonics," Nat. Photonics 3, 55-58 (2008).
60. O. Hess, J. B. Pendry, S. A. Maier, R. F. Oulton, J. M. Hamm, and K. L. Tsakmakidis, "Active nanoplasmonic metamaterials," Nat. Mater. 11, 573-584 (2011).

61. M. Ferrera, N. Kinsey, A. Shaltout, C. DeVault, V. Shalaev, and A. Boltasseva, "Dynamic nanophotonics [Invited]," J. Opt. Soc. Am. B 34, 95-103 (2017)

62. Z. Wang, J. Liu, A. Z. Xu, Y. Xue, L. Jiang, J. Song, F. Huang, Y. Wang, Y. L. Zhong, Y. Zhang, Y. Cheng, and Q. Bao, "Wavelengthtunable waveguides based on polycrystalline organic-inorganic perovskite microwires," Nanoscale 8, 6258-6264 (2016).

63. A. A. Savchenkov, W. Liang, A. B. Matsko, V. S. Ilchenko, D. Seidel, and L. Maleki, "Tunable optical single-sideband modulator with complete sideband suppression," Opt. Lett. 34, 1300-1302 (2009).

64. A. Munir and V. Fusco, "Frequency selective surface with dual band switchable reflection and transmission characteristics," Microwave and Opt. Technol. Lett. 51, 2059-2061 (2009).

65. A. She, S. Zhang, S. Shian, D. R. Clarke, and F. Capasso, "Adaptive metalenses with simultaneous electrical control of focal length, astigmatism, and shift," Sci. Adv. 4, eaap9957 (2018).

66. H. T. Chen, W. J. Padilla, M. J. Cich, A. K. Azad, R. D. Averitt, and A. J. Taylor, "A metamaterial solid-state terahertz phase modulator," Nat. Photonics 3, 148-151 (2009).

67. Y. W. Huang, H. W. Lee, R. Sokhoyan, R. A. Pala, K. Thyagarajan, S Han, D. P. Tsai, and H. A. Atwater, "Gate-tunable conducting oxide metasurfaces," Nano Lett. 16, 5319-5325 (2016).

68. J. Y. Ou, E. Plum, J. F. Zhang, and N. I. Zheludev, "An electromechanically reconfigurable plasmonic metamaterial operating in the near-infrared," Nat. Nanotechnol. 8, 252-255 (2013).

69. R. Singh, A. K. Azad, Q. X. Jia, A. J. Taylor, and H. T. Chen, "Thermal tunability in terahertz metamaterials fabricated on strontium titanate single-crystal substrates," Opt. Lett. 36, 1230-1232 (2011).

70. B. X. Wang, X. Zhai, G. Z. Wang, W. Q. Huang, and L. L. Wang, "Frequency tunable metamaterial absorber at deep-subwavelength scale," Opt. Mater. Express 5, 227-235 (2015).

71. H. T. Chen, J. F. O'Hara, A. K. Azad, A. J. Taylor, R. D. Averitt, D. B. Shrekenhamer, and W. J. Padilla, "Experimental demonstration of frequency-agile terahertz metamaterials," Nat. Photonics 2, 295298 (2008)

72. Y. Zhou, X. Y. Hu, C. Li, H. Yang, and Q. H. Gong, "All-optical tunable dual Fano resonance in nonlinear metamaterials in optical communication range," J. Mod. Opt. 65, 206-212 (2018).

73. N. I. Zheludev and Y. S. Kivshar, "From metamaterials to metadevices," Nat. Mater. 11, 917-924 (2012).

74. J. Valente, J. Y. Ou, E. Plum, I. J. Youngs, and N. I. Zheludev, "A magneto-electro-optical effect in a plasmonic nanowire material," Nat. Commun. 6, 7021 (2015).

75. S. M. Kamali, E. Arbabi, A. Arbabi, Y. Horie, and A. Faraon, "Highly tunable elastic dielectric metasurface lenses," Laser Photon. Rev. 10, 1002-1008 (2016).

76. Z. C. Chen, M. Rahmani, Y. D. Gong, C. T. Chong, and M. H. Hong, "Realization of variable three-dimensional terahertz metamaterial tubes for passive resonance tunability," Adv. Mater. 24, OP143OP147 (2012).

77. H. S. Ee and R. Agarwal, "Tunable metasurface and flat optical zoom lens on a stretchable substrate," Nano Lett. 16, 2818-2823 (2016).

78. Y. H. Fu, A. Q. Liu, W. M. Zhu, X. M. Zhang, D. P. Tsai, J. B. Zhang, T. Mei, J. F. Tao, H. C. Guo, X. H. Zhang, J. H. Teng, N. I. Zheludev, G. Q. Lo, and D. L. Kwong, "A micromachined reconfigurable metamaterial via reconfiguration of asymmetric split-ring resonators," Adv. Funct. Mater. 21, 3589-3594 (2011).

79. W. M. Zhu, A. Q. Liu, T. Bourouina, D. P. Tsai, J. H. Teng, X. H. Zhang, G. Q. Lo, D. L. Kwong, and N. I. Zheludev, "Microelectromechanical Maltese-cross metamaterial with tunable terahertz anisotropy," Nat. Commun. 3, 1274 (2012).

80. W. M. Zhu, A. Q. Liu, X. M. Zhang, D. P. Tsai, T. Bourouina, J. H. Teng, X. H. Zhang, H. C. Guo, H. Tanoto, T. Mei, G. Q. Lo, and D. L. Kwong, "Switchable magnetic metamaterials using micromachining processes," Adv. Mater. 23, 1792-1796 (2011).

81. C. P. Ho, P. Pitchappa, Y. S. Lin, C. Y. Huang, P. Kropelnicki, and C. Lee, "Electrothermally actuated microelectromechanical systems 
based omega-ring terahertz metamaterial with polarization dependent characteristics," Appl. Phys. Lett. 104, 161104 (2014).

82. T. Kan, A. Isozaki, N. Kanda, N. Nemoto, K. Konishi, H. Takahashi, M. Kuwata-Gonokami, K. Matsumoto, and I. Shimoyama, "Enantiomeric switching of chiral metamaterial for terahertz polarization modulation employing vertically deformable MEMS spirals," Nat. Commun. 6, 8422 (2015).

83. E. Arbabi, A. Arbabi, S. M. Kamali, Y. Horie, M. Faraji-Dana, and A. Faraon, "MEMS-tunable dielectric metasurface lens," Nat. Commun. 9, 812 (2018).

84. N. Jiang, Z. Xiaolu, and J. Wang, "Active plasmonics: principles, structures, and applications," Chem. Rev. 118, 3054-3099 (2018).

85. A. Nemati, Q. Wang, M. Hong, and J. Teng, "Tunable and reconfigurable metasurfaces and metadevices," Opto-Electron. Adv. 1, 18000901 (2018).

86. J. Park, J. H. Kang, S. J. Kim, X. G. Liu, and M. L. Brongersma, "Dynamic reflection phase and polarization control in metasurfaces," Nano Lett. 17, 407-413 (2017).

87. A. Forouzmand and H. Mosallaei, "Tunable two dimensional optical beam steering with reconfigurable indium tin oxide plasmonic reflectarray metasurface," J. Opt. 18, 125003 (2016).

88. P. Guo, R. D. Schaller, J. B. Ketterson, and R. P. H. Chang, "Ultrafast switching of tunable infrared plasmons in indium tin oxide nanorod arrays with large absolute amplitude," Nat. Photonics 10, 267-273 (2016).

89. S. Saha, A. Dutta, C. DeVault, V. M. Shalaev, and A. Boltasseva, "Ultrafast tunable metasurface with transparent conducting oxide antenna array," in CLEO: QELS_Fundamental Science, OSA Technical Digest (Optical Society of America, 2018), paper FTh4M.4.

90. M. Ferrera and E. G. Carnemolla, "Ultra-fast transient plasmonics using transparent conductive oxides," J. Opt. 20, 024007 (2016).

91. J. Gu, R. Singh, Z. Tian, W. Cao, Q. Xing, M. He, J. W. Zhang, J. Han, H. Chen, and W. Zhang, "Terahertz superconductor metamaterial," Appl. Phys. Lett. 97, 071102 (2010).

92. I. I. Smolyaninov and V. N. Smolyaninova, "Metamaterial superconductors," Phys. Rev. B 91, 094501 (2015).

93. I. I. Smolyaninov and V. N. Smolyaninova, "High Tc superconducting THz metamaterial for ultrastrong coupling in a magnetic field," ACS Photon. 5, 3977-3983 (2018).

94. A. Tsiatmas, V. A. Fedotov, F. J. García de Abajo, and N. I. Zheludev, "Low-loss terahertz superconducting plasmonics," New. J. Phys. 14, 115006 (2012).

95. N. Lazaridesab and G. P. Tsironis, "Superconducting metamaterials," Phys. Rep. 752, 1-67 (2018).

96. I. I. Smolyaninov and V. N. Smolyaninova, "Metamaterial superconductors," Nanophotonics 7, 795-818 (2018).

97. L. Kang, Q. Zhao, H. Zhao, and J. Zhou, "Magnetically tunable negative permeability metamaterial composed by split ring resonators and ferrite rods," Opt. Express 16, 8825-8834 (2008).

98. H. Zhao, B. Li, J. Zhou, L. Kang, Q. Zhao, and W. Li, "Abnormal refraction of microwave in ferrite/wire metamaterials," Opt. Express 19, 15679-15689 (2011)

99. Y. Zhu, D. S. Antao, R. Xiao, and E. N. Wang, "Real-time manipulation with magnetically tunable structures," Adv. Mater. 26, 64426446 (2014).

100. A. E. Serebryannikov, A. Lakhtakia, and E. Ozbay, "Single and cascaded, magnetically controllable metasurfaces as terahertz filters," J. Opt. Soc. Am. B 33, 834-841 (2016).

101. A. E. Serebryannikov, A. Lakhtakia, and E. Ozbay, "Characteristic attributes of multiple cascaded terahertz metasurfaces with magnetically tunable subwavelength resonators," Ann. Phys. (Berlin) 530, 1700252 (2018).

102. Q. Zhao, L. Kang, B. Du, B. Li, J. Zhou, , H. Tang, and X. Liang, and B. Zhang, "Electrically tunable negative permeability metamaterials based on nematic liquid crystals," Appl. Phys. Lett. 90, 011112 (2007).

103. X. D. Wang, D. H. Kwon, D. H. Werner, I. C. Khoo, A. V. Kildishev, and V. M. Shalaev, "Tunable optical negative-index metamaterials employing anisotropic liquid crystals," Appl. Phys. Lett. 91, 143122 (2007).

104. Q. Liu, Y. Cui, D. Gardner, X. Li, S. He, and I. I. Smalyukh, "Selfalignment of plasmonic gold nanorods in reconfigurable anisotropic fluids for tunable bulk metamaterial applications," Nano Lett. 10, 1347-1353 (2010).

105. K. Stratford, O. Henrich, J. S. Lintuvuori, M. E. Cates, and D. Marenduzzo, "Self-assembly of colloid-cholesteric composites provides a possible route to switchable optical materials," Nat. Commun. 5, 3954 (2014).

106. G. Y. Si, Y. H. Zhao, E. S. P. Leong, and Y. J. Liu, "Liquid-crystalenabled active plasmonics: a review," Materials 7, 1296-1317 (2014).

107. K. Chen, E. S. P. Leong, M. Rukavina, T. Nagao, Y. J. Liu, and Y. Zheng, "Active molecular plasmonics: tuning surface plasmon resonances by exploiting molecular dimensions," Nanophotonics 4, 186197 (2015).

108. Y. Sun, L. Jiang, L. Zhong, Y. Jiang, and X. Chen, "Towards active plasmonic response devices," Nano Res. 8, 406-417 (2015).

109. J. Wang, S. Liu, S. Guruswamy, and A. Nahata, "Reconfigurable liquid metal based terahertz metamaterials via selective erasure and refilling to the unit cell level," Appl. Phys. Lett. 103, 221116 (2013).

110. W. Zhang, Q. Song, W. Zhu, Z. Shen, P. Chong, D. P. Tsai, C. Qiu, and A. Q. Liu, "Metafluidic metamaterial: a review," Adv. Phys. X 103, 221116 (2018).

111. L. Ju, B. Geng, J. Horng, C. Girit, M. Martin, Z. Hao, H. A. Bechtel, X. Liang, A. Zettl, Y. R. Shen, and F. Wang, "Graphene plasmonics for tunable terahertz metamaterials," Nat. Nanotechnol. 6, 630-634 (2011).

112. S. Hoon Lee, M. Choi, T. Kim, S. Lee, M. Liu, X. Yin, H. K. Choi, S. S. Lee, C. Choi, S. Choi, X. Zhang, and B. Min, "switching terahertz waves with gate-controlled active graphene metamaterials," Nat. Mater. 11, 936-941 (2012).

113. J. Kim, H. Son, D. J. Cho, B. S. Geng, W. Regan, S. Shi, K. Kim, A. Zett, Y.-R. Shen, and F. Wang, "Electrical control of optical plasmon resonance with graphene," Nano Lett. 12, 5598-5602 (2012).

114. H. G. Yan, X. S. Li, B. Chandra, G. Tulevski, Y. Q. Wu, M. Freitag, W. Zhu, P. Avouris, and F. Xia, "Tunable infrared plasmonic devices using graphene/insulator stacks," Nat. Nanotechnol. 7, 330-334 (2012).

115. H. Hajian, H. Caglayan, and E. Ozbay, "Long-range Tamm surface plasmons supported by graphene-dielectric metamaterials," J. Appl. Phys. 121, 033101 (2017).

116. A. Fallahi and J. Perruisseau-Carrier, "Design of tunable biperiodic graphene metasurfaces," Phys. Rev. B 86, 195408 (2012).

117. Y. G. Chen, T. S. Kao, B. Ng, X. Li, X. G. Luo, B. Lukýanchuk, S. A. Maier, and M. H. Hong, "Hybrid phase-change plasmonic crystals for active tuning of lattice resonances," Opt. Express 21, 13691-13698 (2013).

118. M. Liu, H. Y. Hwang, H. Tao, A. C. Strikwerda, K. Fan, G. R. Keiser, A. J. Sternbach, K. G. West, S. Kittiwatanakul, J. Lu, S. A. Wolf, F. G. Omenetto, X. Zhang, K. A. Nelson, and R. D. Averitt, "Terahertzfield-induced insulator-to-metal transition in vanadium dioxide metamaterial," Nature 487, 345-348 (2012).

119. H. Kim, N. Charipar, E. Breckenfeld, A. Rosenberg, and A. Piqué, "Active terahertz metamaterials based on the phase transition of VO2 thinfilms," Thin Solid Films 596, 45-50 (2015).

120. H. Liu, J. Lu, and X. R. Wang, "Metamaterials based on the phase transition of VO2," Nanotechnology 29, 024002 (2018).

121. Q. Wang, E. T. F. Rogers, B. Gholipour, C. Wang, G. Yuan, J. Teng, and N. I. Zheludev, "Optically reconfigurable metasurfaces and photonic devices based on phase change materials," Nat. Photonics 10 , 60-65 (2016).

122. C. M. Watts, X. Liu, and W. J. Padilla, "Metamaterial electromagnetic wave absorbers," Adv. Mater. 24, OP98-OP120 (2012).

123. J. Y. Rhee, Y. J. Yoo, K. W. Kim, Y. J. Kim, and Y. P. Lee, "Metamaterial-based perfect absorbers," J. of Electromagn. Waves and Appl. 28, 1541-1580 (2014).

124. M. Keshavarz Hedayati, F. Faupel, and M. Elbahri, "Review of plasmonic nanocomposite metamaterial absorber," Materials 7, 12211248 (2014).

125. P. Yu, L. V. Besteiro, Y. Huang, J. Wu, L. Fu, H. H. Tan, C. Jagadish, G. P. Wiederrecht, A. O. Govorov, and Z. Wang, "Broadband metamaterial absorbers," Adv. Opt. Mater. 7, 1800995 (2018). 
126. S. Ogawa and M. Kimata, "Metal-insulator-metal-based plasmonic metamaterial absorbers at visible and infrared wavelengths: a review," Materials 11, 458 (2018).

127. A. Ghobadi, H. Hajian, B. Butun, and E. Ozbay, "Strong light-matter interaction in lithography-free planar metamaterial perfect absorbers," ACS Photon. 5, 4203-4221 (2018).

128. Y. Ra'di, V. S. Asadchy, and S. A. Tretyakov, "Total absorption of electromagnetic waves in ultimately thin layers," IEEE Trans. Antennas Propag. 61, 4606-4614 (2013).

129. S. A. Tretyakov and S. I. Maslovski, "Thin absorbing structure for all incidence angles based on the use of a high-impedance surface," Microwave and Opt. Technol. Lett. 38, 175-178 (2003).

130. N. Engheta, "Thin absorbing screens using metamaterial surfaces," in IEEE Antennas and Propagation Society International Symposium (IEEE Cat. No. 02CH37313) (IEEE, 2002), Vol. 2.

131. O. Luukkonen, F. Costa, C. R. Simovski, A. Monorchio, and S. A. Tretyakov, "A thin electromagnetic absorber for wide incidence angles and both polarizations," IEEE Trans. Antennas Propag. 57, 3119-3125 (2009).

132. G. V. Naik, V. M. Shalaev, and A. Boltasseva, "Alternative plasmonic materials: beyond gold and silver," Adv. Mater. 25, 3264-3294 (2013).

133. J. Park, J. Kang, X. Liu, and M. L. Brongersma, "Electrically tunable epsilon-near-zero (ENZ) metafilm absorbers," Sci. Rep. 5, 15754 (2015).

134. J. R. Hendrickson, S. Vangala, C. Dass, R. Gibson, J. Goldsmith, K. Leedy, and D. E. Walker, Jr., J. W. Cleary, W. Kim, and J. Guo, "Coupling of epsilon-near-zero mode to gap plasmon mode for flat top wideband perfect light absorption," ACS Photon. 5, 776-781 (2018).

135. M. Morea, K. Zang, T. I. Kamins, M. L. Brongersma, and J. S. Harris, "Electrically tunable, CMOS-compatible metamaterial based on semiconductor nanopillars," ACS Photon. 5, 4702-4709 (2018).

136. J. Kim, E. G. Carnemolla, C. DeVault, A. M. Shaltout, D. Faccio, V. M. Shalaev, A. V. Kildishev, M. Ferrera, and A. Boltasseva, "Dynamic control of nanocavities with tunable metal oxides," Nano Lett. 18, 740-746 (2018).

137. G. R. Keiser, J. Zhang, X. Zhao, X. Zhang, and R. D. Averitt, "Terahertz saturable absorption in superconducting metamaterials," J. Opt. Soc. Am. B 33, 2649-2655 (2016).

138. J. Zhang, J. Tian, and L. Li, "Pantoscopic and temperature-controlled dual-band perfect absorber based on strontium titanate material," Mater. Res. Express 5, 065802 (2018).

139. Y. Bian, C. Wu, H. Li, and J. Zhai, "A tunable metamaterial dependent on electric field at terahertz with barium strontium titanate thin film," Appl. Phys. Lett. 104, 042906 (2014).

140. W. Lia, J. Wei, W. Wang, D. Hu, Y. Li, and J. Guan, "Ferrite-based metamaterial microwave absorber with absorption frequency magnetically tunable in a wide range," Mater. Des. 110, 27-34 (2016).

141. M. C. Ricci, X. Hua, R. Prozorov, A. P. Zhuravel, A. V. Ustinov, and S. M. Anlage, "Tunability of superconducting metamaterials," IEEE Trans. Appl. Supercond. 17, 918-921 (2007).

142. N. K. Grady and B. G. Perkins, Jr., H. Y. Hwang, N. C. Brandt, D. Torchinsky, R. Singh, L. Yan, D. Trugman, S. A. Trugman, Q. X. Jia, A. J. Taylor, K. A. Nelson, and H.-T. Chen, "Nonlinear high temperature superconducting terahertz metamaterials," New J. Phys. 15, 105016 (2013).

143. G. L. Dakovski, W. G. Lee, D. G. Hawthorn, N. Garner, D. Bonn, W. Hardy, R. Liang, M. C. Hoffmann, and J. J. Turner, "Enhanced coherent oscillations in the superconducting state of underdoped YBa2Cu3O6+x induced via ultrafast terahertz excitation," Phys. Rev. B 91, 220506 (2015)

144. B. Wang, X. Zhai, G. Wang, W. Huang, and L. Wang, "Frequency tunable metamaterial absorber at deep-subwavelength scale," Opt. Express 5, 227-235 (2016).

145. J. Hao, Veronique Sadaune, L. Burgnies, and D. Lippens, "Ferroelectrics based absorbing layers," J. Appl. Phys. 116, 043520 (2014).

146. T. Wang, P. Wang, Y. Wang, and L. Qiao, "A broadband far-field microwave absorber with a sandwich structure," Mater. Des. 95, 486-489 (2016).
147. M. Lei, N. Feng, Q. Wang, Y. Hao, S. Huang, and K. Bi, "Magnetically tunable metamaterial perfect absorber," J. Appl. Phys. 119, 244504 (2016).

148. W. Wang, J. Wang, M. Yan, J. Wang, H. Ma, M. Feng, and S. Qu, "Dual band tunable metamaterial absorber based on cuboid ferrite particles," J. Phys. D 51, 315001 (2018).

149. Y. Huang, G. Wen, W. Zhu, J. Li, L. Si, and M. Premaratne, "Experimental demonstration of a magnetically tunable ferrite based metamaterial absorber," Opt. Express 22, 16408-16417 (2014).

150. D. Shrekenhamer, W. Chen, and W. J. Padilla, "Liquid crystal tunable metamaterial absorber," Phys. Rev. Lett. 110, 177403 (2013).

151. M. P. Hokmabadi, A. Tareki, E. Rivera, P. Kung, R. G. Lindquist, and S. M. Kim, "Investigation of tunable terahertz metamaterial perfect absorber with anisotropic dielectric liquid crystal," AIP Adv. 7, 015102 (2017).

152. Z. Yin, Y. Lu, T. Xia, W. Lai, J. Yang, H. Lu, and G. Deng, "Electrically tunable terahertz dual-band metamaterial absorber based on a liquid crystal," RCS Adv. 8, 4197 (2018).

153. A. Komar, R. Paniagua-Domínguez, A. Miroshnichenko, Y. F. Yu, Y. S. Kivshar, A. I. Kuznetsov, and D. Neshev, "Dynamic beam switching by liquid crystal tunable dielectric metasurfaces," ACS Photon. 5, 1742-1748 (2018).

154. W. Li, L. Zhao, Z. Dai, H. Jin, F. Duan, J. Liu, Z. Zeng, J. Zhao, and Z. Zhang, "A temperature-activated nanocomposite metamaterial absorber with a wide tenability," Nano Res. 11, 3931-3942 (2018).

155. M. Keshavarz Hedayati, M. Javaheri, A. U. Zillohu, H. J. ElKhozondar, M. S. Bawa'aneh, A. Lavrinenko, F. Faupel, and M. Elbahri, "Photo-driven super absorber as an active metamaterial with a tunable molecular-plasmonic coupling," Adv. Opt. Mater. 2, 705$710(2014)$

156. J. Park, J. Kang, X. Liu, S. J. Maddox, K. Tang, P. C. McIntyre, S. R. Bank, and M. L. Brongersma, "Dynamic thermal emission control with InAs-based plasmonic metasurfaces," Sci. Adv. 4, eaat3163 (2018).

157. S. S. Mirshafieyan and D. A. Gregory, "Electrically tunable perfect light absorbers as color filters and modulators," Sci. Rep. 8, 2635 (2018).

158. H. Chen, W. J. Padilla, J. M. O. Zide, A. C. Gossard, A. J. Taylor, and R. D. Averitt, "Active terahertz metamaterial devices," Nature 444, 597-600 (2006)

159. N. Shen, M. Massaouti, M. Gokkavas, J. Manceau, E. Ozbay, M. Kafesaki, T. Koschny, S. Tzortzakis, and C. M. Soukoulis, "Optically implemented broadband blueshift switch in the terahertz regime," Phys. Rev. Lett. 106, 037403 (2011).

160. W. Yue, L. Xin, Z. W. Chao, L. Qian, Z. Ying, and M. J. Shou, "Broadband tunable hybridized metamaterials absorber at terahertz regime," Ferroelectrics 528, 38-44 (2018).

161. J. Q. Gu, R. Singh, X. J. Liu, X. Q. Zhang, Y. F. Ma, S. Zhang, S. A. Maier, Z. Tian, A. K. Azad, H.-T. Chen, A. J. Taylor, J. Han, and W. Zhang, "Active control of electromagnetically induced transparency analogue in terahertz metamaterials," Nat. Commun. 3, 1151 (2012).

162. M. R. Shcherbakov, S. Liu, V. V. Zubyuk, A. Vaskin, P. P. Vabishchevich, G. Keeler, T. Pertsch, T. V. Dolgova, I. Staude, I. Brener, and A. A. Fedyanin, "Ultrafast all-optical tuning of directgap semiconductor metasurfaces," Nat. Commun. 8, 17 (2017).

163. H. Hajian, A. Soltani-Vala, and M. Kalafi, "Characteristics of band structure and surface plasmons supported by a one-dimensional graphene-dielectric photonic crystal," Opt. Commun. 292, 149-157 (2013).

164. J. S. Gomez-Diaz, C. Moldovan, S. Capdevila, J. Romeu, L. S. Bernard, A. Magrez, A. M. Ionescu, and J. Perruisseau-Carrier, "Self-biased reconfigurable graphene stacks for terahertz plasmonics," Nat. Commun. 6, 6334 (2015).

165. J. Poumirol, P. Q. Liu, T. M. Slipchenko, A. Y. Nikitin, L. MartinMoreno, J. Faist, and A. B. Kuzmenko, "Electrically controlled terahertz magneto-optical phenomena in continuous and patterned graphene," Nat. Commun. 8, 14626 (2017).

166. Y. D. Kim, Y. Gao, R. Shiu, L. Wang, O. B. Aslan, M.-H. Bae, H. Kim, D. Seo, H.-J. Choi, , S. H. Kim, A. Nemilentsau, T. Low, C. Tan, D. K. Efetov, T. Taniguchi, K. Watanabe, K. L. Shepard, T. F. Heinz, 
D. Englund, and J. Hone, "Ultrafast graphene light emitters," Nano Lett. 18, 934-940 (2018).

167. E. Hendry, P. J. Hale, J. Moger, A. K. Savchenko, and S. A. Mikhailov, "Coherent nonlinear optical response of graphene," Phys. Rev. Lett. 105, 097401 (2010).

168. A. N. Grigorenko, M. Polini, and K. S. Novoselov, "Graphene plasmonics," Nat. Photonics 6, 749-758 (2012).

169. T. Low and P. Avouris, "Graphene plasmonics for terahertz to midinfrared applications," ACS Nano 8, 1086-1101 (2014).

170. H. Hajian, A. Soltani-Vala, and M. Kalafi, "Optimizing terahertz surface plasmons of a monolayer graphene and a graphene parallel plate waveguide using one-dimensional photonic crystal," J. Appl. Phys. 114, 033102 (2013).

171. H. Hajian, A. Soltani-Vala, M. Kalafi, and P. T. Leung, "Surface plasmons of a graphene parallel plate waveguide bounded by Kerr-type nonlinear media," J. Appl. Phys. 115, 083104 (2014).

172. H. Hajian, I. D. Rukhlenko, P. T. Leung, H. Caglayan, and E. Ozbay, "Guided plasmon modes of a graphene-coated Kerr slab," Plasmonics 11, 735-741 (2017).

173. Y. Yao, R. Shankar, M. A. Kats, Y. Song, J. Kong, M. Loncar, and F. Capasso, "Electrically tunable metasurface perfect absorbers for ultrathin mid-infrared optical modulators," Nano Lett. 14, 65266532 (2014).

174. Z. Fang, Y. Wang, A. E. Schlather, Z. Liu, P. M. Ajayan, F. J. G. de Abajo, P. Nordlander, X. Zhu, and N. J. Halas, "Active tunable absorption enhancement with graphene nanodisk arrays," Nano Lett. 14, 299-304 (2014).

175. S. Thongrattanasiri, F. H. L. Koppens, and F. J. G. de Abajo, "Complete optical absorption in periodically patterned graphene," Phys. Rev. Lett. 108, 047401 (2012).

176. R. Alaee, M. Farhat, C. Rockstuhl, and F. Lederer, "A perfect absorber made of a graphene micro-ribbon metamaterial," Opt. Express 20, 28017-28024 (2012).

177. H. Hajian, A. Ghobadi, B. Butun, and E. Ozbay, "Tunable, omnidirectional, and nearly perfect resonant absorptions by a graphene-hBNbased hole array metamaterial," Opt. Express 26, 16940-16954 (2018).

178. X. Wang, X. Jiang, Q. You, J. Guo, X. Dai, and Y. Xiang, "Tunable and multichannel terahertz perfect absorber due to Tamm surface plasmons with graphene," Photon. Res. 5, 536-542 (2017).

179. Y. Chang, C. Liu, C. Liu, S. Zhang, S. R. Marder, E. E. Narimanov, Z. Zhong, and T. B. Norris, "Realization of mid-infrared graphene hyperbolic metamaterials," Nat. Commun. 7, 10568 (2016).

180. H. Hajian, A. Ghobadi, S. Abedini Dereshgi, B. Butun, and E. Ozbay, "Hybrid plasmon-phonon polariton bands in graphene-hexagonal boron nitride metamaterials," J. Opt. Soc. Am. B 34, D29-D35 (2017).

181. H. Kocer, S. Butun, B. Banar, K. Wang, S. Tongay, J. Wu, and K. Aydin, "Thermal tuning of infrared resonant absorbers based on hybrid gold-VO2 nanostructures," Appl. Phys. Lett. 106, 161104 (2015).

182. H. Kocer, S. Butun, E. Palacios, Z. Liu, S. Tongay, D. Fu, K. Wang, J. $\mathrm{Wu}$, and K. Aydin, "Intensity tunable infrared broadband absorbers based on $\mathrm{VO}_{2}$ phase transition using planar layered thin films," Sci. Rep. 5, 13384 (2015).

183. L. Yang, P. Zhou, T. Huang, G. Zhen, L. Zhang, L. Bi, X. Weng, J. $X i e$, and L. Deng, "Broadband thermal tunable infrared absorber based on the coupling between standing wave and magnetic resonance," Opt. Mater. Express 7, 2767-2776 (2017).

184. A. Tittl, A. K. U. Michel, M. Schäferling, X. Yin, B. Gholipour, L. Cui, M. Wuttig, T. Taubner, F. Neubrech, and H. Giessen, "A switchable mid-infrared plasmonic perfect absorber with multispectral thermal imaging capability," Adv. Mater. 27, 4597-4603 (2015).

185. K. V. Sreekanth, S. Han, and R. Singh, " $\mathrm{Ge}_{2} \mathrm{Sb}_{2} \mathrm{Te}_{5}$-based tunable perfect absorber cavity with phase singularity at visible frequencies," Adv. Mater. 30, 1706696 (2018).

186. H. Hajian, A. Ghobadi, A. E. Serebryannikov, B. Butun, G. A. E. Vandenbosch, and E. Ozbay, " $\mathrm{VO}_{2}-\mathrm{hBN}$-graphene-based bifunctional metamaterial for bi-tunable asymmetric transmission and nearly perfect resonant absorption characteristics," J. Opt. Soc. Am. B 36, 1607-1615 (2019).

187. A. Kumar, T. Low, K. H. Fung, P. Avouris, and N. X. Fang, "Tunable light-matter interaction and the role of hyperbolicity in graphenehBN system," Nano Lett. 15, 3172-3180 (2015).

188. H. Hajian, O. Ozbay, and H. Caglayan, "Enhanced transmission and beaming via a zero-index photonic crystal," Appl. Phys. Lett. 109, 031105 (2016).

189. X. Liu and W. J. Padilla, "Dynamic manipulation of infrared radiation with MEMS metamaterials," Adv. Opt. Mater. 1, 559-562 (2013).

190. M. Liu, M. Susli, D. Silva, G. Putrino, H. Kala, S. Fan, M. Cole, L. Faraone, V. P. Wallace, W. J. Padilla, D. A. Powell, I. V. Shadrivov, and M. Martyniuk, "Ultrathin tunable terahertz absorber based on MEMS-driven metamaterial," Microsyst. Nanoeng. 3, 17033 (2017).

191. M. Ghaderi, E. Karimi Shahmarvandi, and R. F. Wolffenbuttel, "CMOScompatible mid-IR metamaterial absorbers for out-of-band suppression in optical MEMS," Opt. Mater. Express 8, 1696-1707 (2017).

192. W. Zhang, Q. Song, W. Zhu, Z. Shen, P. Chong, D. P. Tsai, C. Qiu, and A. Q. Liu, "Metafluidic metamaterial: a review," Adv. Phys.: X 3 , 1417055 (2018).

193. A. Salim and S. Lim, "Review of recent metamaterial microfluidic sensors," Sensors 18, 232 (2018).

194. K. Ling, H. Ki Kim, M. Yoo, and S. Lim, "Frequency-switchable metamaterial absorber injecting eutectic gallium-indium (EGaln) liquid metal alloy," Sensors 15, 28154-28165 (2015).

195. Y. J. Yoo, H. Y. Zheng, Y. J. Kim, J. Y. Rhee, J.-H. Kang, K. W. Kim, H. Cheong, Y. H. Kim, and Y. P. Lee, "Flexible and elastic metamaterial absorber for low frequency, based on small-size unit cell," Appl. Phys. Lett. 105, 041902 (2014). 